SEARCH MARKET EQUILIBRIUM,

\title{
BILATERAL HETEROGENEITY
}

\section{AND REPEAT PURCHASES}

\author{
March 1987 - Revised May 1988 \\ $N^{\circ} 8806$
}

Ro land BENABOU

CEPREMAP

142, rue du Chevaleret

75013 PARIS, France

I am grateful to Patrick Bolton, Peter Diamond, Pierre Picard and Jean Tirole for helpful comments on earlier versions of this paper. All remaining errors are of course mine. 


\title{
SEARCH MARKET EQUILIBRIUM, BILATERAL HETEROGENEITY
}

\section{AND REPEAT PURCHASES}

\author{
A B STRACT
}

This paper develops a general model of a search market with heterogeneity among both buyers (through search costs) and sellers (through production costs). It provides a unifying framework for previous models with unilateral heterogeneity, and is thereby able to simultaneously account for : a) price dispersion which does not rest on the indeterminacy of individual prices; b) active search in equilibrium; $c$ ) entry of firms; d) the matching of sellers with high costs, hence high prices, with buyers whose search costs are also high. These results are then extended from single to repeated purchase markets - possibly with buyer renewal - by embedding the previous model into a dynamic game with incomplete information between sellers and buyers. Stationary equilibria (Bayesian perfect) of this game are shown to be isomorphic, in a certain sense, to single-purchase equilibria, while offering an even more realistic description of a monopolistically competitive search market : an endogenous number of firms charge constant but fully optimal prices, reflecting the interplay of preferences, production costs and search costs ; in every period a flow of consumers enter and invest in a thorough search for a suitable seller, with which they then form a stable long-run relationship until leaving the market.

J.E.L.Classification $n^{\circ}: 022,026$

Keywords : search, price dispersion, monopolistic competition

\section{EQUILIBRE DE PROSPECTION. HETEROGENEITE BILATERALE}

\section{ET ACHATS REPETES}

\section{RESUME}

Ce papier développe un modèle général d'équilibre de prospection qui prend en compte à la fois I'hétérogénéité des acheteurs (à travers leurs coûts de prospection) et celle des vendeurs (à travers leurs coûts de production). Il généralise les modèles préexistant qui confinaient la diversité à un seul côté du marché et permet ainsi de rendre compte à la fois de : a) une dispersion de prix qui ne repose pas sur l'indétermination des prix individuels ; b) une prospection active à l'équilibre; c) l'entrée endogène de nouveaux vendeurs; d) la composition de la clientèle de chaque type de vendeur. On étend ensuite ces résultats au cas d'achats répétés, en incorporant le modèle précédent dans un jeu dynamique à information incomplète entre acheteurs et vendeurs. On montre que les équilibres (Bayésiens parfaits) stationnaires de ce jeu sont isomorphes, en un certain sens, aux équilibres du modèle à achat unique, tout en fournissant une description encore plus réaliste du fonctionnement d'un marché de concurrence monopolistique : un nombre endogène d'entreprises tarifient des prix constants mais reflétant optimalement 1'interaction des préférences, des coûts de production et des coûts de prospection ; à chaque période, de nouveaux acheteurs arrivent sur le marché et investissent dans une recherche approfondie, jusqu'à trouver un vendeur qui leur convienne, avec lequel ils forment alors une relation de long-terme stable jusqu'à leur sortie du marché.

Classification J.E.L. $n^{\circ}: 022,026$

Mots-clef : prospection, recherche, concurrence monopolistique, dispersion de 
INTRODUCTION

Monopolistically competitive markets are characterized by substantial diversity among both sellers and buyers, and often by stable long-run relationships between the two. Sellers with higher costs charge higher prices, and a larger fraction of their customers is composed of buyers who have a higher valuation of time, or more limited alternatives. In the case of repeat purchases, buyers do not search each time for a suitable seller, but rather invest in an initial search to find a long-term supplier.

This paper develops a general model of search market equilibrium with bilateral heterogeneity, for both cases of single and repeat purchases. Its first objective is to account for the above stylized facts ; the second one is to unify previous models of search market equilibrium within a more general theoretical framework ; the third one is to provide a model able to simultaneously explain : a) Price dispersion, reflecting the variety of production and search technologies and their interplay with preferences ; b) active search by buyers, constraining the optimal pricing behaviour of firms; c) determinacy of individual price strategies, leading to the stability of the equilibrium with respect to small perturbations ; d) endogenous entry of firms ; e) robustness of the equilibrium configuration across single and repeated purchase markets, including those in which the population of buyers is renewed over time.

A market where identical buyers search sequentially among identical sellers who can freely set prices is subject to the well known monopoly price result of Diamond [1971]: as long as the search cost is not exactly zero, the unique equilibrium is for all firms to charge the monopoly price. Thus there is 
neither price dispersion nor search, no matter how small the search cost or how many firms there are. The literature on sequential search has remedied this problem by allowing heterogeneity in consumers' search costs (Axe11 [1977], von zur Muehlen [1980], Rob [1985], Stiglitz [1987]), in their tastes (Diamond [19871) or in firms' production costs (Reinganum [1979]). (1) In Bénabou [1986b] it is shown that in fact price dispersion can exist, and search matter, even with identical consumers and firms, provided the frictions in the functioning of the market are not one-sided (consumer search cost) but also affect firms (costly price adjustment in an inflationary environment), even in arbitrarily sma 11 amounts.

Departing from this minimalism, one will introduce here differences among both firms and consumers. Diversity is indeed an important feature of actual markets, and it is therefore not surprising that (as with market frictions) models which confine it to only one set of actors are in a certain way "unbalanced" :

- Models with consumer heterogeneity generate price dispersion and search, but firms are indifferent between the various prices charged in the market. As the equilibrium rests on indeterminacy, it can only arise by chance (if just enough sellers choose to charge each price) ${ }^{(2)}$ and its stability is problematic. An exception is the repeated purchase model of Mc Millan and Morgan [1984], but there, on the contrary, firms are forever stuck by consumer loyalty at their initial prices, which are non-optimal and therefore can again only have arisen by chance.

- The model of firm heterogeneity of Reinganum [1979] does have sellers solving we 11-behaved optimisation problems leading to different prices, but still does 
not fully reconcile search with price dispersion : all firms with cost below a critical level charge their monopoly price, as in Diamond [1971], while those with higher cost bunch at consumers' common reservation price, so that no search takes place in equilibrium. Thus, there is no effect of search on the firms among which price dispersion exists, and conversely, there is no price dispersion among those which are affected by search.

Clearly, the strenghs and weaknesses of these two types of models are complementary. This paper combines both approaches into a general model of twosided heterogeneity ${ }^{(3)}$ which generates equilibria possessing all the desired properties. The methodology and results are presented in terms of consumer search in a good's market, but they are easily recast in terms of job search by heterogeneous workers in a labour market where firms differ by the ir productivities.

Section I presents the general framework and provides a full characterisation of equilibria as solutions to a functional fixed-point equation. Section II is devoted to the case of uniformly distributed search costs, for which existence, stability and uniqueness results are established; moreover, a very simple closed-form solution is derived - as in Mc Minn [1980] - when individual demand in the absence of search is inelastic (II.1), while the Reinganum [1979] model is precisely generalized when it is isoelastic (II.2). Section III provides a general method for easily constructing closed form equilibria with complex cost (of search and production) and price distributions, but simple pricing rules. Section IV examines repeat purchases and customer renewal, extending the previous results in the form of Bayesian perfect equilibria of a dynamic game with both incomplete and imperfect 
information. Most proofs are given in appendix at the end of the paper.

\section{I - THE GENERAL MODEL}

I.1. Firms and consumers : A continuum of firms can produce and sell a homogeneous product with constant returns to scale. Their unit costs are distributed on some $[\underline{c}, \bar{c}]$, where $0<\underline{c}<\bar{c}<+\infty$, with cumulative distribution function (c.d.f.) $G(c)$. There is a continuum of consumers, each of whom has indirect utility function $V(p)\left(V^{\prime}\left\langle 0, V^{\prime \prime}>0\right)\right.$ for buying the firms' product at a price $p$. In an equilibrium, consumers know the distribution of prices in the market, but not the prices charged by individual sellers ; the cumulative distribution function of prices will be denoted by $F(p)$, with support in $[\underline{p}, \bar{p}], \underline{p} \geqslant 0$. Except for the first one, which is free, price quotations are obtained only through visits to sellers, each of which entails a cost to the consumer. This search cost represents the resources expended on each visit (time, transportation), to which could be added the cost of waiting for another try when consumers are impatient. Consumers are identical except for their unit search costs, which are distributed on some $[0, \bar{\sigma}]$, where $\sigma<\bar{\sigma}<+\infty$, with a c.d.f. $Q(\sigma)$ admitting a finite density $q(\sigma)$. Atoms in the distribution of search costs are thus excluded, but this entails no significant loss of generality, since they can be obtained as limits of finite densities (cf. Section [1.2). Similarly, there is no cost in assuming that $a$ is rightcontinuous and that $x(p)=-V^{\prime}(p)$ is positive for all $p$ within the relevant range; $x(p)$ is the consumer's demand (derived from preferences) in the absence of search, or conditional on buying at the price $p$, and will be referred to as his "conditional demand" from here on. 
1.2. Search : There is a single period, during which prices remain fixed and all search takes place. The standard following assumptions are made :

a) consumers' preferences are additively separable into the (indirect) utility derived from the good $V(p)$, and search costs; b) There is no limit to the number of searches which can be conducted. Thus wealth effects from search costs are treated as negligible. Under these two assumptions, the optimal search strategy for a consumer with search cost $\sigma$ is independent of whether or not previous offers can be recalled (recall is never used), and characterized by the reservation price $r$ given by :

$$
V(r)=-\sigma+\int_{0}^{r} V(p) d F(p)+\int_{r}^{\infty} V(r) d F(p)
$$

when a solution to this equation exists. It expresses indifference between accepting an offer of $r$ and pursuing search with the same decision rule, which yields an expected utility of $V(r)$. Equivalently:

$$
\begin{gathered}
a=\int_{0}^{r}(V(p)-V(r)) d F(p)=\int_{0}^{r}-V^{\prime}(p) F(p) d p \text { or }: \\
r_{F}(r) \equiv \int_{0}^{r} \times(p) F(p) d p=0
\end{gathered}
$$

by Roy's identity. The function $r_{F}$ is continuous and strictly increasing on $[\underline{p},+\infty)$, hence it has an inverse, denoted $R_{F}$, on $\left(0, \Gamma_{F}(+\infty)\right)$. For 0 in this interval, (2) has a unique solution $r=R_{F}(\sigma)$. For $a>r_{F}(+\infty)$, (2) has no solution as an equality : the search cost is so high that any offer is preferable to search. For these consumers, define $r=R_{F}(\sigma)=+\infty$. Thus we have for all $0 \geq 0$ :

$$
\left.R_{F}(\sigma)=\sup \left\langle r \in R_{+}\right| \Gamma_{F}(r)=\int_{0}^{r} x(p) F(p) d p \leqslant a\right\}
$$

The subscript $F$ will be dropped from $r_{F}$ and $R_{F}$ when no confusion is possible. 
Note from (3) that an upward shift in conditional demand $x(p)$ lowers $r$ for any consumer : a more desirable good involves a larger purchase at any given price and therefore justifies more search.

1.3. Demand : Denote by $1 / \theta$ the endogenous density of operating firms (per consumer) in the market. The demand curve facing firms will now be derived by aggregating the individual search rules of all consumers, as in Bénabou [1986a], [1987]. Consider first consumers with search cost $\sigma<\Gamma(+\infty)$. By (2), the density $v(r)$ of reservation prices in this population is :

$$
v(r)=(q \circ \Gamma)(r) \Gamma^{\prime}(r)=(q \circ \Gamma)(r) F(r) \times(r)
$$

Consumers with reservation price in $[r, r+d r]$ each have a probability $F(r)$ of being successful in any single search. By the law of large numbers, each firm is therefore visited by $\theta v(r)$ dr of them on their first search, $\theta v(r)(1-F(r))$ on their second search, $\theta v(r)(1-F(r))^{k-1}$ on their $k$-th search, etc., hence a total of $\theta v(r) / F(r) d r=\theta(q \circ \Gamma)(r) \times(r) d r$ individuals with $R_{F}(\sigma) \in[r, r+d r]$. Summing all those who accept an offer of $p$, plus the non-searching individuals $(r=+\infty$, or $\sigma>r(+\infty))$ yields the number $s_{F}(p)$ of consumers who buy from any firm charging a price $p$ :

$$
\left.s_{F}(p)=\theta<\int_{D}^{\infty}(q \circ \Gamma)(r) \times(r) d r+\int_{\Gamma(+\infty)}^{\infty} q(a) d \sigma\right)
$$

Since each buyer purchases $x(p)$ units, the demand curve facing each $f$ irm is:

$$
\left.D_{F}(p)=\theta \times(p)<\int_{D}^{\infty}(q \circ \Gamma)(r) \times(r) d r+\int_{\Gamma(+\infty)}^{\infty} q(\sigma) d \sigma\right)
$$

Contrary to the formula used by Axe11 [1977], $D(p)$ is not simply the product of $x(p)$ and the demand curve which obtains when conditional demand is inelastic; this is because preferences also enter into the determination of 
the optimal reservation price (cf. equation (3)). The properties of this demand function will now be examined. First, define $\bar{r}=R(\bar{\sigma})$ and $\underline{r}=R(\sigma)$, respectively the highest and lowest reservation prices (possibly infinite) in the population. Demand is zero for $p>\bar{r}$, positive and decreasing below. For $p<\underline{r}$, the bracketed term in $(5)$ is independent of $p$, so that $D(p)$ is simply proportional to a monopolist's demand curve $x(p)$, as in Reinganum [1979]. Finally, $D_{F}$ is almost everywhere differentiable, and its kinks correspond to the left discontinuities of q (Stiglitz [1987] focused in particular on the kink at $r$ resulting from $q(0)>0)$. Its elasticity is :

$$
e_{F}(p)=-\frac{p x^{\prime}(p)}{x(p)}+\frac{(q \circ \Gamma)(p) x(p) p}{\int_{p}^{\infty}(q \circ \Gamma)(\Gamma) \times(r) d \Gamma+\int_{\Gamma(+\infty)}^{\infty} q(\sigma) d \sigma}
$$

i.e. the sum of the elasticity resulting from preferences, and that resulting from search, so that firms' pricing problem may be we 11 behaved even with very inelastic conditional demand (cf. Section II.1).

1.4. Pricing rules : Since no consumer buys above $\bar{r}$, only those firms with $c \leqslant \bar{r}$ can operate profitably. Hence :

$$
1 / \theta=G(\bar{r})=\left(G \circ R_{F}\right)(\bar{\sigma})
$$

These firms maximize the continuous function $\pi(p, c) \equiv(p-c) D_{F}(p)$ over $[c, \bar{r}]$, where $D_{F}(p)$ is given by (5). For $c<\bar{r}$, any solution $p *$ must clearly be interior; thus, if $\pi$ is twice differentiable at $p *$, the usual first and second-order conditions must be satisfied :

$$
C_{F}\left(p^{*}\right)=c, \quad C_{F}^{\prime}\left(p^{*}\right) \geqslant 0
$$

where $C_{F}(p) \equiv p\left(1-1 / C_{F}(p)\right)$ is marginal revenue (with respect to output) as a function of price. By (6), the right-continuous function $C_{F}$ is defined for 
$p \in(0, \bar{r})$ by :

$$
c_{F}(p)=p-\left[\frac{(q \circ \Gamma)(p) \times(p)}{\int_{p}^{\infty}(q \circ \Gamma)(r) \times(r) d r+\int_{\Gamma(+\infty)}^{\infty} q(\sigma) d \sigma}-\frac{x^{\prime}(p)}{x(p)}\right]^{-1} .
$$

When $\pi$ is not twice differentiable at $p^{*}$, similar conditions apply, involving left and right limits of $C_{F}$ (cf. proof of Theorem I).

\subsection{Equilibrium : For $F$ to be an equilibrium distribution, it must coincide} with the distribution which arises when each operating firm with cost c charges a solution $p(c)$ to $(9)$. By $(9), C_{F}\left(0^{+}\right)<0$ and $C_{F}\left(\bar{r}^{-}\right)=\bar{r}$. (7) Suppose for the moment that $C_{F}$ is increasing and continuous (the usual assumption of a marginal revenue decreasing continuously with the quantity sold). Then (8) defines for al1 $c<\bar{r}$ a unique optimal price $p_{F}(c)=C_{F}^{-1}(c)$. For all $p$, let $e_{x}(p)=$ $-p x^{\prime}(p) / x(p) ;$ by $(9), p\left(1-1 / e_{x}(p)\right)<C_{F}(p)<p$, with equality if and only if, respectively, $p<\underline{r}$ and $p=\bar{r}$. Hence ${ }^{(8)}$ :

$$
c<p_{F}(c)<p^{m}(c)
$$

(where $p^{m}(c) \&+\infty$ is the firm's monopoly price), with equality if and only if, respectively, $c=\bar{r}$ and $c<C_{F}(r)$. Finally, since $C_{F}$ is increasing, the resulting price distribution is given by ${ }_{\mathrm{F}}$ below :

Definition : For any $F: R_{+}+R_{+}$, define $r_{F}$ as in (2), $C_{F}$ as in (9), $\bar{r}_{F}=\sup \left\{r \geqslant 0\left|r_{F}(r)<\bar{\sigma}\right\rangle\right.$, and $\phi_{F}: R_{+} \rightarrow[0,1]$ by :

$$
\begin{cases}{ }_{F}(p)=\frac{G\left(C_{F}(p)\right)}{G\left(\bar{r}_{F}\right)} & \text { for } p<\bar{r} \\ { }_{F}(p)=1 & \text { for } p \geqslant \bar{r}_{F} .\end{cases}
$$

More generally, by restricting attention to "well-behaved" equilibria, in which 
firms' profit functions do not possess multiple local maxima (i.e. are strictly quasi-concave) one can ensure that $C_{F}$ is non-decreasing (possibly discontinuous, where $q$ is) over $\left\{p \mid C_{F}(p) \in\right.$ support $\left.(G)\right\}$; the above line of reasoning then remains valid and leads to following characterization result.

Theorem I : Let the distribution $G$ of firm costs be continuous. A function $F: R_{+}+R_{+}$is an equilibrium price distribution where almost all firms' profit functions are strictly quasi-concave if and only if :

a) $F$ is a fixed point of the functional mapping ${ }^{\prime} F \rightarrow{ }^{\circ}$.

b) $F$ is non-decreasing.

The equilibrium is then generated by each firm with cost be low $\bar{r}_{F}$ charging the (almost always) unique solution $p_{F}(c)$ to $C_{F}\left(p^{-}\right) \leqslant c<C_{F}\left(p^{+}\right)$, while the others (if any) do not operate. Consumers search with reservation prices $R_{F}(\sigma)$.

Proof : cf. appendix.

The standard monopoly pricing problem is characterized by the conjunction of the familiar first-order condition, $p=1 /\left(1-1 / e_{x}(p)\right)$, which can be viewed as a one-dimensional fixed-point problem, and of a monotonicity condition which ensures that the profit function is locally concave. Theorem 1 in fact generalizes these fixed point and monotonicity conditions to the infinite dimensional space of functions from $R_{+}$into itself. Interestingly, the global monotonicity condition (b) on $F$ is equivalent (given (a)) to the local monotonicity of marginal revenue $C_{F}(p)$ at (almost) every firm's solution to its first order condition. If $G$ were discontinuous, the possibility of a positive mass of firms' being indifferent between several prices would lead to a similar characterization of equilibrium distributions as fixed points of a 
functional correspondence.

While Theorem I fully characterizes (well behaved) equilibria, and provides a basis to search for them numerically, it does not address the question of existence; as can be seen from the combination of (2), (9) and (11) it is in general a nearly insoluble one. In particular, with an endogenous demand function $D_{F}$, marginal revenue $C_{F}$ need not be increasing (in price) for all F (cf. (9)), so that does not generally map increasing functions into themselves. (10) The conditions which guarantee existence and/or uniqueness in the standard monopoly problem can therefore not be assumed here, but have to be derived (as equilibrium properties) from assumptions about the two cost distributions and consumer preferences; this will be done in Sections II and III.

\subsection{Discussion : Figure 1 describes the essential features of a typical}

equilibrium. Firms with cost above $\bar{r}_{F}$ cannot retain any consumer at a profitable price and therefore stay out of the market. Firms with cost below $c_{0} \equiv c_{F}\left(r_{F}^{-}\right)$can charge their monopoly price $\left(p_{F}(c)=p^{m}(c)\right)$ without inducing any consumer to leave, while all others are constrained by search to price below the ir monopoly level $\left(c<p_{F}(c)<p^{m}(c)\right)$. Conversely, it is the latters' prices (for $c>c_{1}=r_{F} \geqslant c_{0}$ ) which trigger active consumer search.

A discontinuity in $q$ at some point $\sigma$ (in the case of Figure 1 , at 0 ) causes a similar discontinuity in $C_{F}$ at $R_{F}(\sigma)$ (here at $r_{f}$ ), leading to a bunching of prices by a whole segment $\left[c_{0}, c_{1}\right]=\left[C_{F}\left(\bar{r}_{F}\right), C_{F}\left(\bar{r}_{F}\right)\right]$ of firms; equivalently, each of these firms' price is insensitive to cost variations in the $\left[c_{0}, c_{1}\right]$ range (cf. Stig]itz [1987] for a discussion of this "rigidity"). The combination of this bunching with the monopolistic behaviour of firms with 


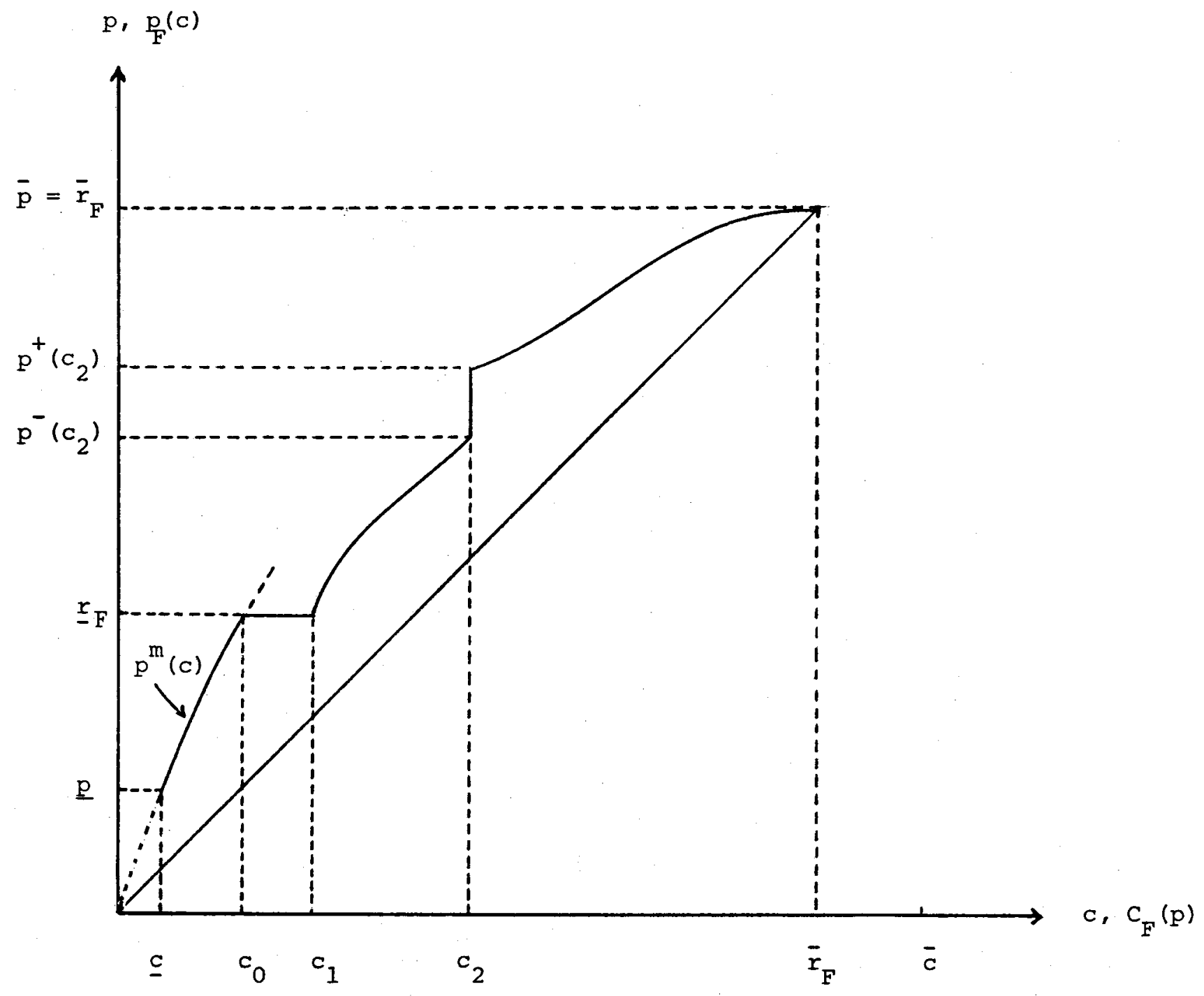

Figure 1: The marginal revenue function $C_{F}(p)$ and the optimal pricing rule $\mathrm{p}_{\mathrm{F}}(\mathrm{c})$. 
cost $c \leqslant c_{0}=c_{F}\left(r_{-F}\right)$ is the central feature of the Reinganum [1979] mode]. Conversely, a flat section in $C_{F}(p)$ corresponds to a multivalued optimal price $\left[p^{-}\left(c_{2}\right), p^{+}\left(c_{2}\right)\right]$ at a point like $c_{2}$ on Figure 1 . If the cost distribution happens to have an atom at a point like $c_{2}$, then a positive mass of firms are indifferent between all prices in $\left[p_{2}^{-}(c), p_{2}^{+}(c)\right]$; this indeterminacy is the central feature of models with identical firms such as those of Axe1] [1977], von zur Muehlen [1980], Rob [1985] or Stiglitz [1987]. For instance, to obtain the differential equation which the search cost density q must satisfy in order for an equilibrium with continuous dispersion to exist with identical firms $(\underline{c}=\bar{c})$, as in the last two of these references, it suffices to equate $(p-c) D_{F}(p)$ - as defined by (5) - to a constant, and require that $F$ be non-decreasing from zero to one.

Discontinuities or flat sections in $C_{F}$ are non-generic, limiting cases ; when the distributions $q$ and $G$ are slightly perturbed, the equilibrium reverts to a single-valued, continuous and increasing function associating to each cost type $c$ the corresponding optimal price $p_{F}(c)$. In the sequel, the subscript $F$ will be dropped from $r_{F}, \bar{r}_{F}, p_{F}$, etc.. when no confusion is possible.

1.7. Job search : The above methodology, and all the paper's results, are directly applicable to a job search problem. Let workers derive utility $V(w)\left(V^{\prime}(w) \equiv x(w)>0, V^{\prime \prime}(w)<0\right)$ from a wage $w$, firms have constant marginal productivities of labor distributed according to $G(\lambda)$ and offer wages distributed according to $F(w)$. It then essentially suffices to replace in the preceding formulas $V(p)$ by $V(w)$ and, since the reservation wage is now a minimum acceptable offer, $\left(G(c), F(p), \phi_{F}(w)\right)$ by $\left(1-G(\lambda), 1-F(w), 1-\phi_{F}(w)\right)$, 
$\Gamma_{F}(+\infty)$ by $\Gamma_{F}(0)$, and to transpose the bounds of integration $\int_{r}^{\infty}$ into $\int_{0}^{r}$ (and vice versa) in any integral over $d F(p)$. Thus for instance, reservation prices are determined by :

$$
R_{F}(\sigma)=\inf \left(r \in R_{+} \mid \Gamma_{F}(r)=\int_{r}^{\infty} x(w)(1-F(w)) d w<\sigma\right)
$$

( $\Gamma_{F}$ is now decreasing), and firms face the labor supply curve :

$$
\left.S_{F}(W)=\theta \times(W)<\int_{0}^{W}(q \circ \Gamma)(r) \times(r) d r+\int_{\Gamma(0)}^{\infty} q(\sigma) d \sigma\right\}
$$

with $1 / \theta=(1-G)\left(R_{F}(\bar{\sigma})\right)$, and so on.

\section{II - UNIFORMLY DISTRIBUTED SEARCH COSTS}

In this section, we assume :

$$
(\forall \sigma \in[\underline{\sigma}, \bar{\sigma})) \quad(q(\sigma)=1 /(\bar{\sigma}-\underline{\sigma}))
$$

1I.1. Ine lastic conditional demand :

To show how the elasticity from search alone can generate an equilibrium with price dispersion, assume that $x(p)=1$ for all $p$ case, which is treated here as a natural application of Theorem I, has in fact been examined previously by Mc Minn [1980]. While the characterization of the equilibrium derived here (independently) is similar to his, the two sets of results turn out to be complementary : existence, stability and (conditions for) uniqueness of equilibrium are formally established here, while he focuses on the comparative statics of price dispersion with respect to search costs, assuming existence (in the free entry case). Under the above assumptions, $\Gamma(+\infty)=\int_{0}^{\infty} F(p) d p=+\infty$, since $\lim _{p \rightarrow \infty} F(p)=1$, and the demand curve (5) becomes (for $p \leqslant r)$ : 


$$
D(p)=\frac{\theta}{\bar{\sigma}} \int_{\max (p, r)}^{\bar{r}} d r=\frac{\theta}{\bar{\alpha}}(\bar{r}-\max (p, \underline{r}))
$$

For any distribution of prices in the market, firms therefore face a piecewise linear demand curve, leading to an optimal price, for $c<\bar{r}$, of :

$$
p(c)=\max \left\{\frac{1}{2}(c+\bar{r}), \underline{r}\right\rangle
$$

As was shown in section 1.6, the bunching which may occur at $r$ is an artefact due to the discontinuity of the uniform distribution at its lower bound $0=0$; it would be smoothed out if q were continuous. To avoid such a degeneracy, attention will from now on be focused on equilibria (if any) which do not involve bunching (13), i.e. such that the firms with the lowest costs find it optimal to charge a price greater than $\underline{r}: c_{1} \equiv C_{F}(r)<\underline{c}$, cf. Figure 1. Then : $\left(14^{\prime}\right)$

$$
p(c)=\frac{1}{2}(c+\bar{r}), \text { for all c } \bar{r} \text {, }
$$

which does not involve any bunching, and indeed coincides with the optimal rule (14) for all firms with $c \in[\underline{c}, \bar{r}]$, because $\underline{a}=0$ implies that $\underline{r}=\underline{p}$, hence $C(r)=2 p-\bar{r}=2 p(c)-\bar{r}=\underline{c}$. Denote by $E_{F}($.$) and E_{G}($.$) expectations with$ respect to the distributions $F$ and $G$ respectively. Since $\bar{p}<\bar{r}$, (2) yields:

$$
\bar{\sigma}=\int_{0}^{\bar{r}} F(p) d p=\int_{0}^{r}(\bar{r}-p) d F(p)=\bar{r}-E_{F}(p) .
$$

Each firm therefore charges :

$$
p(c)=\frac{1}{2}\left(c+E_{F}(p)+\bar{\sigma}\right)=\frac{1}{2}\left(c+E_{G}(c \mid c \leqslant \bar{r})\right)+\bar{\sigma}
$$


which clearly expresses the monopolistically competitive nature of the equilibrium. Finally, the functional fixed point requirement (11) on $F$ becomes:

$$
F(p)=\min \left(\frac{G(2 p-\bar{r})}{G(\bar{r})}, 1\right\}, \text { with } \bar{r}=r^{-1}(\bar{\sigma})
$$

which reduces to an implicit equation in $\bar{r}$ :

$$
\bar{\sigma}=\int_{0}^{\bar{r}} \frac{G(2 p-\bar{r})}{G(\bar{r})} d p=\frac{1}{2} \int_{0}^{\bar{r}} \frac{G(c)}{G(\bar{r})} d c
$$

since $G$ has support in $R_{+}$; equivalently, defining $H(r)=\int_{0}^{r} G(c)$ dc :

$$
H(\bar{r}) / G(\bar{r}) \equiv \int_{0}^{\bar{r}} \frac{G(c)}{G(\bar{r})} d c=2 \bar{\sigma}
$$

II.1.1. Existence and stability : An equilibrium (with no bunching) will now be shown to be stable, under a reasonable adjustment process, if and only if the curve $H / G$ cuts the horizontal $2 \bar{\sigma}$ from below at that point. Assume that this condition is satisfied, and suppose that $\bar{r}_{0}$ is slightly above the equilibrium value $\bar{r}$. Then $(H / G)\left(\bar{r}_{0}\right)>2 \bar{\sigma}$, or by $\left(14^{\prime \prime}\right)-(15), r_{F} 0\left(\bar{r}_{0}\right)>\bar{\sigma}$, where $F^{0}$ is the distribution associated to $\bar{r}_{0}$ by (15). Consumers with search cost (close to) $\bar{\sigma}$ then adjust their reservation price to $\bar{r}_{1}<\bar{r}_{0}$. Faced with a reduction in demand, expensive firms cut their prices (or even close down) resulting in a lower price distribution $F^{1}$. Subsequently all reservation prices fall, and $\bar{r}_{1}$ decreases to $\bar{r}_{2}$, forcing more firms to cut prices, and so on until the decreasing sequence $(\bar{r})$ has converged to $\bar{r}$. If $(H / G)$ is decreasing in a rightneighborhood of $\bar{r}$, on the contrary, then $r_{F} O\left(\bar{r}_{0}\right)<\bar{\alpha}$ and the adjustment process involves less search, higher prices, more firms, and a sequence $(\bar{r})$ which increases away from $\bar{r}$. A similar reasoning holds for $\bar{r}_{0}<\bar{r}$; therefore $\bar{r}$ is stable if and only if $(H / G)$ is increasing in its neighborhood. 
Theorem II.1 : Let consumers have perfectly inelastic conditional demand, and search costs uniformly distributed on $[0, \bar{\sigma}]$. For any distribution of firm costs $G(c)$, there exists a stable no-bunching equilibrium. Any such equilibrium corresponds uniquely to a maximum consumer reservation price $\vec{r}$ solution to : $(H / G)(\bar{r})=2 \bar{\sigma}$ (with the intersection occuring from below), and results from each firm with cost $c \leqslant \bar{r}$ charging:

$$
p(c)=\frac{1}{2}\left(c+E_{G}(c \mid c \leqslant \bar{r})\right)+\bar{o}
$$

while no firm with cost $c>\bar{r}$ operates. The resulting price distribution is :

$$
F(p)=\min \left\{\frac{G(2 p-r)}{G(\bar{r})}, 1\right\}
$$

and consumers search with reservation prices $R(\sigma)$ given by : $\sigma=\int_{0}^{R(\sigma)} F(p)$ dp.

Proof : cf. appendix.

No matter how complex the distribution of production costs (including atoms, "holes" etc.) and the resulting distribution of prices in the market, the equilibrium pricing rule is extremely simple : each firm charges the half-sum of its own cost and the average cost of all firms in the market, plus a constant measuring search costs. When $\bar{\sigma}$ decreases, both the number of profitable firms and the prices they charge decrease, with only the most efficient firms remaining and behaving competitively $(c=\underline{c}=p(c))$ in the limit, as $\bar{\sigma}$ goes to zero. Mc Minn [1980] shows that price dispersion is a nondecreasing function of $\bar{\sigma}$, provided the cost distribution $G$ has a decreasing density; this condition is also one of the cases covered by Theorem II.1.2 below, which guarantees that the equilibrium is unique. 
II.1.2. Uniqueness : The solution to $(16)$ is unique, for all values of $\bar{\sigma}>0$, if and only if $H / G$ is increasing, or $G / H$ decreasing on $[c,+\infty)$; note that this requires the c.d.f. G to be continuous on this interval.

Theorem 11.2 : The no-bunching equilibrium is unique for all $\bar{\sigma}$ if and only if the integral of $G: H(r)=\int_{0}^{r} G(c)$ dc has a decreasing hazard rate $H^{\prime} / H=G / H$, on $I \equiv[\underline{c},+\infty)$. A sufficient condition is that the distribution $G$ itse if have a continuous density $G^{\prime}$ and a decreasing hazard rate $G^{\prime} / G$.

Proof : cf. appendix.

Any distribution with non-increasing density, and many usual distributions such as (on any support) the uniform, normal, exponential, Pareto, logistic, or extreme value, have a decreasing hazard rate, and therefore generate a unique, stable, equilibrium without bunching.

\section{II.2. Isoe lastic conditional demand}

Search alone can thus generate we11-behaved demand curves and price dispersion, even when conditional demand is very inelastic. The dual case of (constant) $e_{x}>1$ highlights the interplay of search and preferences in the determination of the equilibrium, and provides an exact generalization of Reinganum's [1979] model to the case where consumers are heterogeneous. In addition, the number of firms in the market is endogenized. In particular, it confirms her conjecture that this would lead to increased price dispersion accompanied by active consumer search.

Let therefore $V(p)=p^{1-\alpha} /(\alpha-1)$ with $\alpha>1$, and $\sigma$ be distributed uniforly on $[\underline{a}, \bar{\sigma}], 0<\underline{\sigma}<\bar{\sigma}$. The equilibrium demand curve (5) becomes, for $p \leqslant \bar{r}$ : 
(19)

$$
D(p)=\frac{\theta p^{-\alpha}}{\bar{\sigma}-\underline{\sigma}}\left(\int_{\max (p, r)}^{\Gamma} r^{-\alpha} d r+\max (\bar{\sigma}-\Gamma(+\infty), 0)\right) \text {, or : }
$$

$$
\begin{cases}D(p)=A p^{-\alpha}\left(p^{1-\alpha}-\bar{r}^{1-\alpha}+\beta\right) & \text { for } p \in[\underline{r}, \bar{r}] \\ D(p)=A p^{-\alpha}\left(r^{1-\alpha}-\bar{r}^{1-\alpha}+\beta\right) & \text { for } p<\underline{r}\end{cases}
$$

where $1 / A=(\alpha-1)(\bar{\sigma}-0) / \theta$ and $\beta=(\alpha-1) \max (\bar{\sigma}-\Gamma(+\infty), 0\}$. Note that $\beta>0$ requires $\bar{r}=+\infty$ while, by $(3), \beta=0$ implies $\Gamma(\bar{r})=\bar{\sigma}$; thus one can also write : $\beta=(\alpha-1) \max (\bar{\sigma}-\Gamma(\bar{r}), 0)$. Equation (19) shows how search both increases the elasticity of the original demand curve $x(p)=p^{-\alpha}$, and shifts it vertically - down if $\bar{\sigma}$ is not so large as to result in an infinite reservation price $\bar{r}$, up otherwise. The general formula (9) yields marginal revenue :

$$
C(p)=\left\{\begin{array}{l}
p\left(1-\frac{1}{\alpha}\right) \text { for } p \leqslant \underline{r} \\
C^{*}(p \mid \bar{r}, \beta) \equiv p\left(1-\frac{1}{\alpha}\right)\left(1+\frac{1}{2 \alpha-1-\alpha\left(\bar{r}^{1-\alpha}-\beta\right) p}\right) \text { for } p \in[\underline{r}, \bar{r}]
\end{array}\right.
$$

Lemma 1 : For all $(\alpha, \beta, \bar{r}, c)$ with, $\alpha>1, \beta>0,0 \leqslant \bar{r} \leqslant+\infty$ and $\beta / \bar{r}=0$, the function $p \rightarrow C^{\star}(p \mid \bar{r}, \beta)$ is continuous and increasing from $[0, \bar{r}]$ into itself; its inverse will be denoted as $c+p^{*}(c \mid \bar{r}, \beta)$. The function $p \rightarrow C(p)$ shares the same properties, except for a discontinuity at $\underline{r}$.

Proof : cf. Appendix.

From Theorem I, each firm with cost $c<\bar{r}$ must charge the unique solution to the first-order-condition $C\left(p^{-}\right)<c<c\left(p^{+}\right)$(the second order condition in ( 8 ) is always satisfied because $C$ is increasing). The optimal pricing rule is therefore : 
(21a) - Firms with cost $c<r(1-1 / \alpha)$ price as monopolists: $p(c)=c /(1-1 / \alpha)$;

(21b) - Firms with cost $c \in\left[r(1-1 / \alpha), c^{*}(r \mid \bar{r}, \beta)\right]$ bunch at $p(c)=\underline{r}$;

(21c) - Firms with cost $c \in\left(C^{*}(r \mid \bar{r}, \beta), \bar{r}\right]$ price as search-constrained monopolistic competitors: $p(c)=p *(c \mid \bar{r}, \beta)$;

(21d) - Firms with cost $c>\bar{r}$ (if any) do not operate.

The resulting price distribution is :

(22)

$$
\begin{cases}F(p)=\frac{G(p(1-1 / \alpha))}{G(\bar{r})} & \text { for } p<\underline{r} \\ F(p)=\frac{G\left(C^{*}(p \mid \bar{r}, \beta)\right)}{G(\bar{r})} & \text { for } p \in[\underline{r}, \bar{r}] \\ F(p)=1 & \text { for } p \geqslant \bar{r},\end{cases}
$$

from which consumers search with reservation prices $R(\sigma)$ given by :

$$
R(\sigma)=\sup \left\{r \in R_{+}\left|\int_{0}^{r} p^{-\alpha} F(p) d p \leqslant \sigma\right\rangle .\right.
$$

Finally, the functional fixed point requirement closing the model takes the form $R(\underline{\sigma})=\underline{r}, R(\bar{\sigma})=\bar{\sigma}$, where $R($.$) is given by (23). Replacing F$ by its expression (22) and remembering that $\beta$ is itself a function of $F$, hence of $r$ and $\bar{r}$, leads to the characterisation of an equilibrium as a solution $(r, \bar{r}, \beta)$ to the following fixed-point system :

(24a) $r=\sup \left(r \geqslant 01 \int_{0}^{r} p^{-\alpha} \cdot \frac{G((1-1 / \alpha) p)}{G(\bar{r})} d p \leqslant \underline{a}\right\rangle$ (24b) $\bar{r}=\sup \left\langle r \geqslant 01 \int_{r}^{r} p^{-\alpha} \cdot \frac{G\left(C^{*}(p \mid \bar{r}, \beta)\right)}{G(\bar{r})} d p \leqslant \bar{\sigma}-\underline{\alpha}\right\rangle$ (24c) $\beta=(\alpha-1) \max \left\{0, \bar{\sigma}-\int_{0}^{r} p^{-\alpha} \cdot \frac{G((1-1 / \alpha) p)}{G(\bar{r})} d p-\int_{\Gamma}^{-} p^{-\alpha} \cdot \frac{G\left(C^{*}(p / r, \beta)\right)}{G(\bar{r})} d p\right\}$ 
Theorem 11.3 : Let consumers have isoelastic conditional demand $x(p)=p^{-\alpha}$, $\alpha>1$, and search costs uniformiy distributed on $[0, \bar{\sigma}]$, with $0<0<\bar{\sigma}$. For any distribution of firm costs $G(c)$ with $c>0,{ }^{(15)}$ there exists a stable equilibrium. Any such equilibrium corresponds uniquely to a triplet $(r, \bar{r}, \beta)$ solution to the system $(24 a)-(24 c)$, where $\underline{r}$ and $\bar{r}$ represent respectively the lowest and highest reservation prices in the population, and $\beta /(\alpha-1)$ the mass of consumers with infinite reservation price $(\beta=0$ if $\bar{r}<+\infty)$. The corresponding equilibrium distribution of prices is then (22) and results from the optimal pricing rule (21a)-(21d) and the optimal search rule (23). Proof : cf. appendix.

Such an equilibrium is illustrated in Figure $2 a$. The more efficient firms $(c<c<\underline{r}(1-1 / \alpha))$ can charge their monopoly price, because it is too low for any consumer to reject. Less efficient firms $(r(1-1 / \alpha)<c<\bar{r})$ are increasingly constrained by search, which forces their optimal price further and further down below their monopoly price; at $c=\bar{r}$, the markup has totally disappeared, and less efficient firms cannot operate profitably. At the point $p=\underline{r}$ where search becomes binding, i.e. where consumers start to leave, the demand curve has a (concave) kink, leading to the same price $r$ for a range of costs $\left[r(1-1 / \alpha), C^{*}(r)\right]$ and generating an atom in the distribution of prices. ${ }^{(16)}$ As was shown in Section 1.6 , this results from the discontinuity of the uniform density $q$ at $a$, and would be smoothed out with a continuous one. The pricing behaviour of firms in $\left[\underline{c}, c^{*}(\underline{r} \mid \bar{r}, \beta)\right]$ corresponds exactly to the case treated by Reinganum [19791, while the others are effectively competing through active consumer search. Indeed, the Reinganum model and results - fixed number of firms, identical consumers, no search in equilibrium 


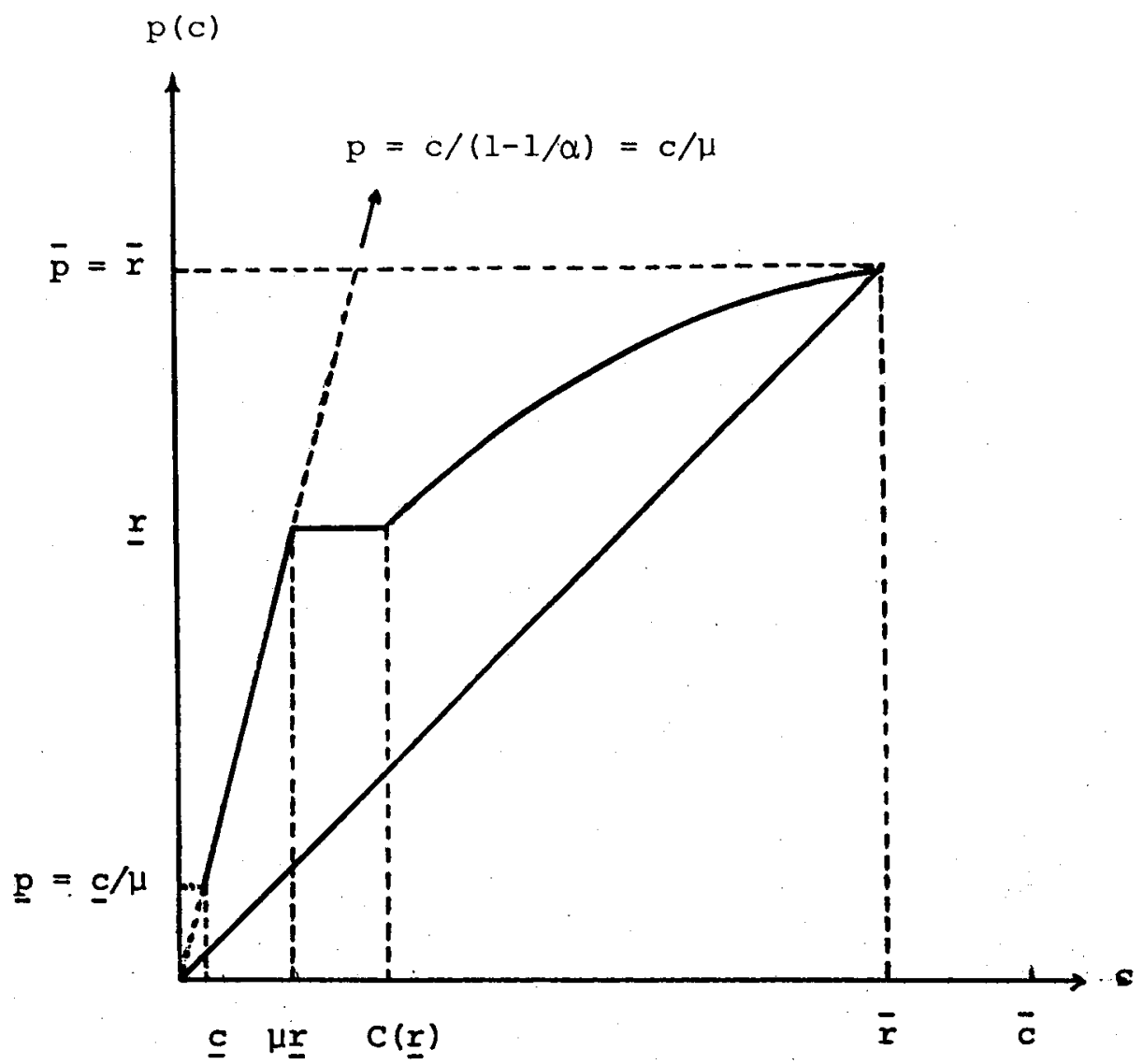

Figure 2a

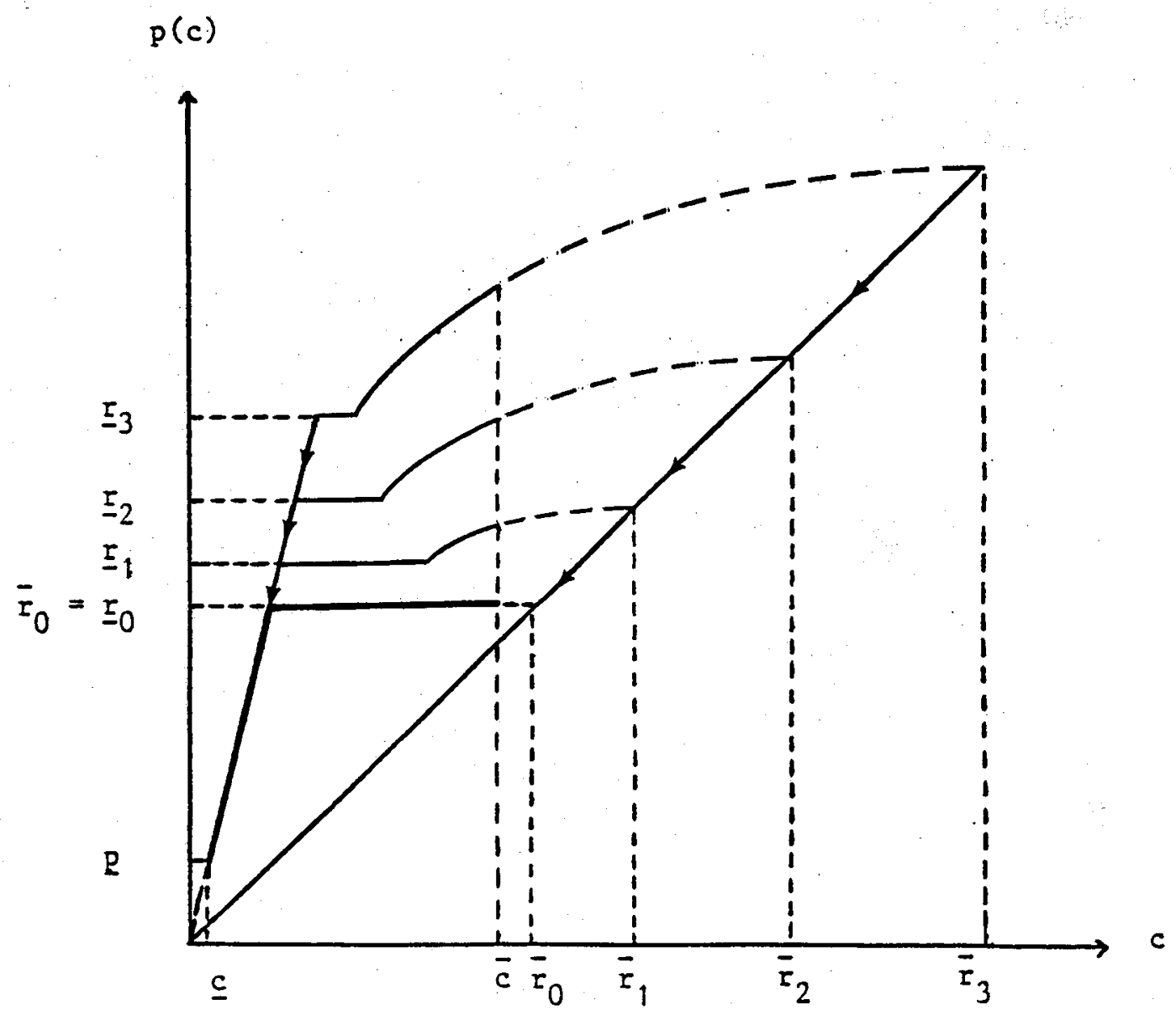

Figure 2b 
- are easily obtained as a limiting case, when $\bar{\sigma}-\underline{\sigma}$ (hence also $\bar{r}-\underline{r}$ )

decreases to zero in equations $(24 a)-(24 c)$ and $(21 a)-(21 d)$ (with the additional constraint $G(\bar{r})=1$ : all firms operate). Figure $2 b$ illustrates this convergence for the case where $\bar{\sigma}$ decreases to a fixed $\underline{0}$.

Each firm in the market creates an externality for others (and consumers) by altering the distribution of prices, so that multiple equilibria may arise. Unlike that of Section 11.1, the equilibrium fixed point problem is here too complicated to derive general conditions guaranteeing uniqueness. One will focus instead on whether there exists an equilibrium in which all firms operate $(G(\bar{r})=1)$ as in Reinganum [1979]. As intuition suggests, such will be the case if search costs are "high enough" with respect to production costs.

Theorem II.4 : There exists an equilibrium with all firms in [c, $\bar{c}]$ operating, if : (i) $\int_{0}^{c} p^{-\alpha} G((1-1 / \alpha) p) d p \leqslant \underline{\sigma}$, or if : (ii) $\int_{\underline{c}}^{\bar{c}} p^{-\alpha} G(p) d p<\bar{\sigma}$

Proof : cf. appendix.

Condition (i) in fact ensures that an equilibrium exists with $\bar{r} \geqslant \underline{r} \geqslant \bar{c}$. It is implied by Reinganum's [1979] assumption that $\bar{c}(1-1 / \alpha)<\underline{c}$; in her case $\bar{r}=r>\bar{c}$. If $(i)$ does not hold but $(i i)$ does, there exists an equilibrium with $+\infty \geqslant \bar{r}>\bar{c}>\underline{r}>\underline{c}$.

\section{III - CONSTRUCTING EQUILIBRIA WITH SIMPLE PRICING RULES}

Under no distribution of search costs other than the uniform is the fixed-point problem (11) solvable for an arbitrary distribution G. But, given some $G($ and $x$ ), it is possible to find search cost distributions leading to an equilibrium with a simple pricing rule, such as : 
(25)

$$
p(c)=\frac{c+b}{\mu} ; \mu>0
$$

If firms face an equilibrium demand curve of the type :

$$
D(p)=k(\max (p, r)+b \alpha)^{-\alpha}
$$

with $\alpha \equiv \mid /(1-\mu)>1$ and $b \geqslant 0$, then (25) indeed generates an equilibrium where all firms participate $(\theta=1)$, provided $\underline{a}=0$, so that $p(c) \geqslant p(c)=\underline{p}=\underline{r}$ for all $c \geqslant c$. Assume inelastic conditional demand for simplicity. Then $\Gamma(+\infty)=+\infty$ and by $(5)$, demand is of the required form (26) if :

$$
(g \circ r)(r)=k^{\prime}(r+b \alpha)^{-(1+\alpha)} \text { for all } r \geqslant \underline{r}
$$

From (2) and (26):

$$
\begin{aligned}
\Gamma(r) & =\int_{0}^{r} F(p) d p=\int_{0}^{r}(G \circ C)(p) d p=\int_{0}^{r} G(\mu p-b) d p \\
& =\mu^{-1} \int_{-b}^{\mu r-b} G(c) d c=\mu^{-1} \int_{0}^{\mu r-b} G(c) d c=\mu^{-1} H(\mu r-b)
\end{aligned}
$$

where, as previously, $H$ is the integral of $G$, which is continuous and increasing from $[(\underline{c},+\infty)$ into $[0,+\infty)$. Therefore :

$$
\Gamma^{-1}(\sigma)=\mu^{-1}\left(b+H^{-1}(\mu \sigma)\right)
$$

maps $[0,+\infty)$ into $[(c+b) / \mu,+\infty)=[\underline{r},+\infty)$, and the solution to (27) is :

$$
q(\sigma)=K\left(\alpha b+H^{-1}(\mu \sigma)\right)^{-(1+\alpha)} \text {, for allo } \sigma 00 \text {. }
$$

Such a function is continuous, constant on $[0, c / \mu]$ and then decreasing. Since $H^{-1} \cdot(\mu \sigma)>\underline{c}+\mu \sigma$, it is summable if and only if $\alpha b+c>0$. Hence : 
Theorem III : Let consumers have ine lastic conditional demand. For any distribution of firm costs $G(c)$, if the density of consumer search costs on $[0,+\infty)$ is:

$$
q(\sigma)=\left[\alpha b+H^{-1}(\mu \alpha)\right]^{-(1+\alpha)} / \int_{0}^{\infty}\left[\alpha b+H^{-1}(\mu y)\right]^{-(1+\alpha)} d y
$$

where $\alpha>1, \mu=1-1 / \alpha, \min (b, c)>0$ and $H$ is the integral of $G$, then there exists an equilibrium where all firms operate and charge $p(c)=(c+b) / \mu$. The resulting price distribution is $F(p)=G(\mu p-b)$ on $[(c+b) / \mu,+\infty)$, and consumers search with reservation prices $R(\sigma)$ given by : $\sigma=\int_{0}^{R(\sigma)} F(p) d p$.

The usefulness of this result in constructing equilibria with search and continuous price dispersion is best illustrated by a couple of examples.

Example 1: Let $G(c)=1-1 / c^{2}$ on $[1,+\infty)$ and, for all $0 \geqslant 0$ :

$$
q(\sigma)=k\left[\sigma+2 \mu+\left(\sigma^{2}+4 \mu \sigma\right)^{1 / 2}\right]^{-(1+\alpha)}
$$

with $\mu=1-1 / \alpha$ in $(0,1)$ and $K$ normalized so that $q$ sums to 1 . In equilibrium, $D(p)=k(\max (p, 1 / \mu))^{-\alpha}, p(c)=c / \mu, F(p)=1-1 / \mu^{2} p^{2}$ on $[1 / \mu,+\infty)$.

Example 2: Let $G(c)=\operatorname{th}(c / 2)=\left(e^{c}-1\right) /\left(e^{c}+1\right)$ on $(0,+\infty)$ and, for all $0 \geqslant 0$ :

$$
q(\sigma)=K\left\{\alpha b+\operatorname{Ln}\left[2 e^{\mu \sigma}-1+2 e^{\mu \sigma / 2}\left(e^{\mu \sigma}-1\right)^{1 / 2}\right]\right\}^{-(1+\alpha)}
$$

with the same notation as above, and $b>0$. In equilibrium, $D(p)=k(p+\alpha b)^{-\alpha}$, $p(c)=(c+b) / \mu$, and $F(p)=\operatorname{th}((\mu p-b) / 2)=\left(e^{\mu p-b}-1\right) /\left(e^{\mu p-b}+1\right)$ on $(b / \mu,+\infty)$.

\section{IV - REPEAT PURCHASE EQUILIBRIUM}

IV.1. Permanent population of consumers

Many goods, especially non-durables, are consumed and purchased repeatedly. Buyers do not search each time for a suitable seller, but rather invest in an initial search to find a permanent or long-term supplier. Similar 
behaviour prevails in the labor market, where job search is followed by a long term implicit or explicit contractual relationship; recall, at this point, that the methodology and results presented here in terms of consumer search for a commodity could equivalently be cast in terms of job search by workers (cf. Section 1.7). Implicit in the above behaviour is some inference of future prices on the basis of past ones : if yesterday's price was acceptable (respectively, too high), it can be expected that today's and tomorrow's will also be acceptable (respectively, too high), and consumers can (resp. can not), economize on search costs by coming back to the same seller in the following period. The type of search costs considered here correspond to resources required to find out about a seller (where he is located, whether the good he sells is indeed the desired one and of appropriate quality, and what price he charges), i.e. "inspection costs" rather than expenses incurred every time a visit or a purchase is made (trips or ordering costs).

The aim of this section is to formalize the inference and decision processes of agents in a repeat purchase market in order to account for such long-term relationships and examine their consequences on equilibrium prices. Mc Millan and Morgan [1984] examine such a market, where identical firms charge constant, different prices, and consumers purchase repeatedly and loyally. However, these results arise in their model through pricing behaviour which is only partly optimal and realistic : in some all-important first period, identical firms somehow distribute themselves appropriately among prices which yield different levels of profits, hence are suboptimal ; consumers then allocate themselves among sellers, and repeat purchases in effect lock firms forever into their initial heterogeneity of clientele and price. This lock-in occurs because : a) a firm cannot profitably lower its 
price after the first period, as competitors' clients would never learn about it ; b) the allocation of consumers resulting from the initial distribution of prices is such that a price increase would cause a loss of consumers outweighing the gain realized on those who stayed. Thus the first period, with its arbitrary prices, looms excessively large, especially since there generally exists an infinity of such equilibria. Moreover, none of those involving price dispersion is likely to be stable to small perturbations (firms have no reason to return to the equilibrium prices), nor robust to the entry of new consumers in the market, which gives firms a chance to correct their initial prices. The class of models presented here, on the other hand, can be extended to repeat purchases in a game theoretic framework, generating more realistic and fully optimal equilibria.

\section{IV.1.1. Description of the game :}

Strategies and Payoffs. Time is discrete and firms set prices in every period (no committment is possible). They will be indexed by $f \in[0,1]$, with associated cost $c^{f}$ and price $p_{t}^{f}$ in period $t$. Buyers live forever, derive in each period a utility $V(p)$ from purchasing the good at a price $p$, and share with firms a discount factor of $\delta<1$. Since $Q$ is assumed to be atomless, they will be indexed by their search cost o $\in[\equiv[\underline{\sigma}, \bar{\sigma}]$. Buyers initially search randomly (search is instantaneous, i.e. there is no limit on the number of searches which can be carried out within one period). In every following period, they decide whether to return to any previously encountered seller, or to search again randomly. Given any price quotation generated by this decision, they decide whether to buy or to continue searching, among previous suppliers 
or randomly. At the beginning of the first period they receive one price quotation for free; all others, which must be obtained through search, entail a cost of a. In every subsequent period, consumers may costlessly return to any previously visited store, or search again, with the same unit cost o as before.

The cost or absence of cost of returning to a previously visited seller turns out to be important and to have complex consequences. If there are positive return costs, buyers' decision is different when they are faced with a given price in their search and when they consider whether or not to go back to a seller believed to charge that same price. For instance, a consumer who is indifferent between accepting some reservation price $R(\sigma)$ and searching, will not return to a firm believed to be charging $R(\sigma)$ if this entails a positive cost. As shown by Bagwe 11 [1985] in a monopolistic context, firms' incentives to fool customers into coming back and then exploit them may then result in complex, non-stationary price strategies. Incorporating such behavior into the general model presented here would be too difficult. It is assumed instead, as mentioned above, that returning to any previously visited seller is costless (as in Mc Millan and Morgan [1984]). This, however, raises a more subtle issue which has to do with how many firms a consumer returns to, off the equilibrium path; it will be discussed at the end of section IV.1.2.

Information structure. When setting its price, a firm has not yet observed how many previous clients have decided to come back and check its price again, nor does it know the prices charged by its competitors. Similarly -these decisions are simultaneous- consumers must decide whether or not to return to a previously visited seller before having observed its current price. They also 
do not know its cost type, and must form beliefs about it as we 11 . Thus they have both incomplete (about firms' types) and imperfect (about unobserved past and/or present prices) information.

IV.1.2. Equilibrium : The equilibrium concept used is that of Bayesian perfect equilibrium (BPE; cf. Harsanyi [1968], Fudenberg and Tirole [1987], [1988]) : at any information set a player's strategy must be optimal given his beliefs, and these beliefs must be obtained by updating priors, using Bayes' rule, the history of observed moves, and the strategies of all other players' types. Refinements such as sequential equilibrium (Kreps and Wilson [1982]) are not available for games with continua of types and actions, but it will still be possible to check that off-the-equilibrium-path beliefs are "reasonable".

To simplify the problem, note first that when looking for equilibrium paths of Bayesian perfect equilibria, attention can be restricted to the specification of strategies and beliefs for histories of the game which do not involve any simultaneous deviations by a positive mass of players (cf. Gul, Sonnenschein and Wilson [1986]). (18) Secondly, in the ir search, return and buying decisions, consumers now compare not only current prices but expected present values of price paths. To keep the analysis tractable, attention will be restricted to equilibria with stationary orice paths (as in Mc Millan and Morgan [1984]); this condition also avoids a Folk-theorem type multiplicity of equilibria. Note that only the equilibrium outcome, and not allowable price strategies, is being restricted here. The (stationary) distribution of equilibrium prices will again be denoted by $F(p)$. Consumers' initial decision problem thus reduces to a once and for all search between the present values of constant price paths, for which the appropriate reservation 
price is (by (3)) :

$$
R_{F}^{\delta}(\sigma) \equiv R_{F}(\sigma(1-\delta))=\sup \left\langle r \geqslant 0 \quad \mid \int_{0}^{r} F(p) \times(p) d p \leqslant \sigma(1-\delta)\right\rangle .
$$

By the optimality principle, they will keep buying (as initially planned) from any firm thus selected as long as its price remains constant, i.e. forever on the equilibrium path. Denote by $Q^{\delta}: \sigma \in[\rightarrow Q(\sigma /(1-\delta))$ the distribution of scaled-down search costs, and $q^{\delta}$ the corresponding density. Any constant price strategy then generates a demand per period $D_{F}^{\delta}(p)$ given by $(5)$, with a replaced by $q^{\delta}$. If firm $f^{\prime}$ s optimal price strategy is a constant $p^{f}$, then in particular $p^{f}$ must maximize $\pi_{F}^{\delta}(p)=(p-c) D_{F}^{\delta}(p) /(1-\delta)$. Therefore :

Theorem IV.1 : Along the equilibrium path of any repeat purchase BPE with stationary prices : (i) Each consumer o initially searches with reservation price $R_{F}^{\delta}(\sigma)$ given by $(31)$, then keeps buying from the same firm in all subsequent periods ; ( $i i)$ Each firm $f$ charges $p_{F}^{\delta}\left(c^{f}\right)$, its optimal price in an equilibrium of the one-shot purchase market for production and search costs distributions $G(c)$ and $Q_{\delta}(\sigma) \equiv Q(\sigma /(1-\delta))$.

The converse result is established in Theorem IV.2 below : every oneshot purchase equilibrium (such as those described in Sections I to III) can be transformed into the outcome of a BPE of the repeat purchase game, in which prices on the equilibrium path (but not outside) are stationary. This result is more difficult to prove, because off the equilibrium path, firms and consumers may have quite complicated strategies ; in particular, the latter may not just buy loyally or search at random, but also return to previously visited firms. Intuitively, the equilibrium works as follows : i) consumers interpret any upward deviation of a firm's price as a signal that its cost is higher than 
previously thought; firms' equilibrium strategies then leads these consumers to view this price increase as permanent. Those with low reservation prices then leave and attach themselves to some other seller, thereby effectively depriving the deviator from any incentive to lower its price again. ii) On the contrary, any downward deviation of the price leaves consumers' beliefs unaffected and is therefore viewed (rightly so) as temporary. Thus price cuts do not ensure repeat purchases, while price hikes result in irreversible customer loss, so that both types of deviations (from $p_{F}^{\delta}\left(c^{f}\right)$ ) are unprofitable. In order to formally establish this result, some definitions are required. Let $H_{t}^{f}$ and $H_{t, n}^{a}$ denote respectively the information sets of $f$ in period $t$, and of $a$ just after his n-th search (or return) step of period $t . H_{t}^{f}$ consists of the firm's past prices $\left\langle p_{s}^{f} \mid s<t\right\rangle$ and experienced demands $\left.\left\langle D_{s}^{f}\right| s<t\right\} . H_{t, n}^{0}$ consists of the "addresses" $I_{t, n}^{0}$ of all previously visited firms and for each of them, the history of prices observed there - in particular the highest price $P_{t, n}^{\sigma}$ (f) ever observed at each of them. The subsets $J_{t, n}^{\sigma}=\left\langle f \in I_{t, n}^{\sigma}\right| P(t, n)$ $<R_{F}^{t}(\sigma)$ ) and $K_{t, n}^{\sigma}=\operatorname{argmin}\left(P_{t, n}^{0}(f) \mid f \in J_{t, n}^{\sigma}\right)$ are thus also part of the information available at $H_{t, n}^{\sigma}$.

Theorem IV.2 : Let $G(c)$ and $Q(a)$ define production and search cost distributions such that $G(c)$ and $Q^{\delta}(\sigma) \equiv Q(\sigma /(1-\delta))$ generate an equilibrium of the one-shot search market, with increasing pricing rule $p_{F}^{\delta}(c)$ and associated price distribution $F(p)$. The following strategies and beliefs constitute a Bayesian perfect equilibrium with stationary path :

(i) Each firm $f$ with cost $c^{f} \leqslant \bar{r}_{F}$ initially charges $p_{0}(c)=p_{F}^{\delta}(c)$; at any information set $H_{t}^{f}(t \geqslant 1)$, it expects all consumers who bought there in the previous period to visit it again first in the current one, and charges: 


$$
\tilde{p}\left(H_{t}^{f}\right)=\max \left\langle p_{F}^{\delta}(c), \max ^{f}\left(p_{s}^{f}|0<s<t-1\rangle\right)\right.
$$

(ii) At any information set $H_{t, n}^{\sigma}$, consumer o has beliefs distributed according to $G(c)$ and $F(p)$ over the cost type and price of firms $f^{\prime} \notin I_{t, n}^{0}$. He believes (with probability one) each firm $f \in I_{t, n}^{\sigma}$ to be of cost type $C_{F}\left(P_{t, n}^{\sigma}(f)\right)$ and to have charged $P_{t, n}^{0}(f)$ in all periods $s<t$ in which he did not visit it. His strategy is given by the following program:

(a) If $j_{t, n}^{\sigma}=\varnothing$, he samples a firm at random, then updates $H_{t, n}^{\sigma}$ into $H_{t, n+1}^{\sigma}$ (b) If $J_{t, n}^{\sigma} \neq \varnothing$, he returns to any firm $f \in K_{t, n}^{\sigma} \neq \varnothing$ and buys from it if:

$$
\left.p_{t}^{f}<\min \left\langle P_{t, n}^{\sigma}\left(f^{\prime}\right)\right| f^{\prime} \in J_{t, n}^{o} \backslash\{f\rangle\right\}
$$

where the r.h.s. is taken to mean $R_{F}^{\delta}(\sigma)$ when $J_{t, n}^{\sigma} \backslash(f)=\varnothing$. If (33) does not hold, he pursues the program after updating $H_{t, n}^{\sigma}$ into $H_{t, n+1}^{\sigma}$, and in particular: $P_{t, n+1}^{\sigma}(f)=\max \left(p_{t}^{f}, P_{t, n}^{\sigma}(f)\right)$;

$J_{t, n+1}^{\sigma}=J_{t, n}^{\sigma}$ or $j_{t, n}^{\sigma} \backslash(f)$, according to $p_{t}^{f}\left\langle R_{F}^{\delta}(\sigma)\right.$;

$k_{t, n+1}^{\sigma}=k_{t, n}^{\sigma}$ or $k_{t, n+1}^{\sigma} \backslash(f)$, according to $p_{t}^{f}\left\langle\min \left\langle P_{t, n}^{0}\left(f^{\prime}\right) \mid f^{\prime} \in J_{t, n}^{0} \backslash(f)\right\rangle\right.$;

Proof : cf. appendix.

A consumer thus goes back to the firm (if any) which he expects to have the lowest price $P_{t, n}^{\sigma}(f)<R_{F}^{\delta}(\sigma)$, and buys there is its actual price $p_{t}^{f}$ is still the lowest he knows of (and below his reservation price). On the equilibrium path, firms charge constant prices and buyers purchase repeatedly from the same store. The stationarity of firms' and consumers' dynamic problems plays, together with the latter's beliefs, an essential role in this 
type of equilibrium. Contrary to Bagwe 11's [1985] model of introductory pricing, for instance, firms have no incentive to misrepresent their cost by initially charging low prices, because the consumers who were fooled into coming back and discovered a high price would leave without buying, and search for another permanent supplier, expecting (rightly) the current one to continue charging high prices in the future. Indeed, let a firm raise its price from $p$ to $p+\varepsilon$; customers with reservation price $r \in[p, p+\varepsilon)$ now believe it to have cost $C_{F}(p+\varepsilon)$ and expect it to charge (forever in the future) the corresponding optimal price, equal to $p+\varepsilon$ by $(32)$; since $\frac{V(p+\varepsilon)}{1-\delta}<V(p+\varepsilon)+\delta \frac{V(r)}{1-\delta}<\frac{V(r)}{1-\delta}$, they prefer to leave rather than remain patrons in the future, or even buy from the firm before resuming search for future purchases. Two important sources of the difference with Bagwe 11 [1985] are the possibility of costless return and consumers' infinite horizon, which together make their decision problem stationary, eliminating in particular any last period in which they could be exploited (19).

While all players' strategies are optimal - on or off the equilibrium path - given their beliefs, close scrutiny of this BPE reveals that buyers use in fact a weakly dominated strategy (w.d.s.). Consumer $\sigma$, after returning to the firm $f \in J_{t, n}^{0}$ which he expects to have the lowest price, buys from $f$ if its actual price $p_{t}^{f}$ is lower than the prices $p_{t, n^{0}}\left(f^{\prime}\right)$ he expects at the other firms he knows of (and lower than $R_{F}^{\delta}(\sigma)$ ). This decision to purchase is expected-utility maximizing, but weakly dominated by the following one : visiting all known firms to find out (at zero cost) their actual price before deciding from which one to purchase. The same criticism applies to Mc Millan and Morgan [1984]. This point, however, is relatively minor, for three 
reasons. First, the players using a w.d.s. are not those who do the signalling, i.e. not firms but consumers ; the usual restriction of out-of-equilibrium beliefs by elimination of strategies which are weakly dominated for certain signalling types is thus not applicable here. Secondly, the beliefs of consumers off the equilibrium path (after witnessing a price change) which sustain their w.d.s. are very reasonable ; in particular, they satisfy Tirole and Fudenberg's [1988] more restrictive definition of BPE : a) a deviation by any player ( $f$ irm) affects beliefs about his type only; b) beliefs obey Baye's rule not just on the equilibrium path, starting from priors, but also starting from the new beliefs triggered by any deviation, and until the next zeroprobability event occurs. Finally, if consumers did keep informed of the prices of all previously visited firms, the latter's strategies would become much more complicated, since lost customers could now be "recalled" by lowering the price. If an equilibrium existed with non stationary price paths, it would involve consumers repeatedly switching between firms, coming back, etc, and would be intractable. On the other hand, if the modified consumer and firm strategies still led to an equilibrium with stationary price paths, it would have the same outcome ( $c f$. Theorem IV.1) as the above BPE, so that the latter's use of a w.d.s. would then be of very minor significance.

IV.1.3. Repeat purchase and price dispersion : The relevant search cost when comparing constant price paths is $\sigma(1-\delta)$. Consumers thus have lower reservation prices, and initially search more than for a single purchase (one can think of local residents versus tourists). They are in fact investing in a thorough search, so as to benefit from a low price and save search costs in all future 
periods. As a result, prices are lower, and the amount of price dispersion smaller, the more frequently the good is purchased (the closer $\delta$ is to one).

\section{IV.2. Repeat purchases with consumer renewal}

The type of equilibrium presented here differs from that of Mc. Millan and Morgan [1984] in two important and related aspects : a) it involves optimal prices in every period; b) as a result, it can accomodate a renewal of the consumer population, which would destroy any equilibrium in which firms are stuck at suboptimal prices. As before, let firms charge constant prices, but assume now that consumers leave the market, or "die" with a constant probability \& $\in(0,1]$ per period ; a constant "number" (mass) $\lambda \geqslant 0$ of new ones enter the market or "are born" in every period. Let the initial (stock) density of consumers at time zero equal its long-run value $\lambda / \mu$; equivalently, one could consider the market as having been in operation since $t=-\infty$. All cohorts are identical in terms of tastes and distribution of search costs, hence also (since they face the same distribution F) of reservation prices. Along the equilibrium path, a firm therefore has the same optimal price with respect to each cohort, i.e. there is no incentive for intergenerational price discrimination (19); neither is there any incentive to fool consumers into coming back, because in that case they will leave without buying (given the appropriate beliefs). These arguments suggest that the same type of BPE path as in Theorems IV.1-IV.2 will still prevail, with consumers searching only upon entering the market. The formal proof is substantially more complicated, because off the equilibrium path there generally are incentives to discriminate between generations : whereas a firm who had deviated and charged $p>p_{F}^{\delta}\left(c^{f}\right)$ would previously not have benefited from lowering back its price lequation 
(32)), it now has conflicting incentives to maintain it because its existing customer base is composed of high-reservation price individuals, and to lower it in order to restore the optimal mix of consumers corresponding to its cost type, by attracting and retaining new clients. The following lemma establishes that there exists an optimal price strategy for any previous sequence of prices, and gives bounds for this strategy.

Lemma 2 : Assume that almost all firms $f$ charge $p_{F}^{\delta \mu}(c)$ in every period, and that consumers (once in the market) have the same strategies as in Theorem IV.2, except that $\delta$ is replaced by $\delta \mu$. For a firm $f$ at any information set $H_{t}^{f}$, the present value of profits to be expected from any sequence of prices $\left\langle p_{s} \mid s \geqslant t\right\rangle$ is then :

$\left.\left.(34) \pi\left(\left\langle p_{s}\right| s>t\right) \mid H_{t}^{f}\right)=\theta \lambda \sum_{s=t}^{+\infty} \delta{ }_{s}^{s-t}\left(p_{s}-c^{f}\right) \sum_{\tau=0}^{+\infty}(1-\mu)^{\tau} D^{\delta \mu}\left(\max \left\langle p_{k}\right| s-\tau \leqslant k \leqslant s\right\}\right)$

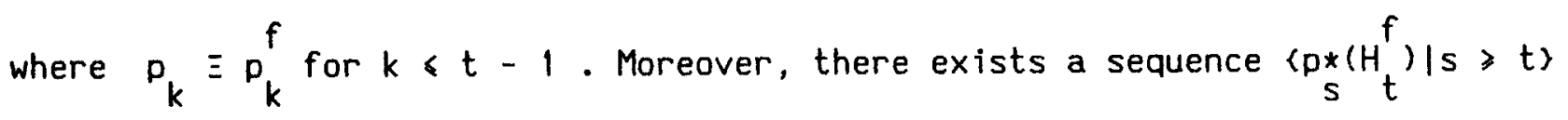
maximizing this present value, with :

$$
\left.\left.(\forall s \geqslant t): p_{F}^{\delta \mu}(c)<\underset{s}{f} t H_{t}^{f}\right) \leqslant \max _{F} \delta \mu\left(p^{f}\right), \max \left(p_{k}^{f} \mid k \leqslant t-1\right)\right) .
$$

Proof : cf. appendix.

Note that if $p_{F}^{\delta \mu}\left(c^{f}\right)$ was charged in the past, it remains optimal in the future. Moreover, the lemma shows that $f$ will never cut its price below $p_{F}^{\delta \mu}\left(c^{f}\right)$ in an attempt to attract new customers : those with $R_{F}^{\delta \mu}(\sigma)<p_{F}^{\delta \mu}(c)$ can never be exploited since they leave permanently as soon as $p$ is raised above $R_{F}^{\delta \mu}(a)$. Lemma 2 also serves as the foundation for the following result. 
Theorem IV. 3 : The results of Theorems IV.1 and IV.2, showing the equivalence (one to one mapping) between stationary paths of repeat purchase Bayesian perfect equilibria and equilibria of the one-shot market, still hold in the presence of buyer renewal, provided that $\delta$ is replaced by $\delta \mu$ and firms price strategy (32) by $p^{*}: H_{t}^{f} \rightarrow p^{*}\left(H_{t}^{f}\right)$ defined in Lemma 2.

Proof : cf. appendix.

The type of equilibrium described in this theorem provides a comprehensive and realistic description of a heterogeneous, monopolistically competitive search market : in every period, consumers come in, invest in an initial search for a suitable seller - of which they then become loyal customers until leaving the market - while an endogenous number of firms charge constant, fully optimal prices reflecting the interplay of preferences, production costs, and search costs. (22)

\section{CONCLUSION}

This paper developed a general model of search market equilibrium with diversity among both sellers and buyers, which is able to account for many empirical facts (price or wage dispersion, active search, allocation of types of buyers across types of sellers, stable clienteles in a repeat purchase context). By unifying and "smoothing out" previous models of unilateral heterogeneity, it shed light on the sources of their unsatisfactory features (indeterminacy, absence of search, bunching), and showed how these could be eliminated as nongeneric, limiting cases. Finally, it extended the single purchase, or static results, to the case of repeated purchases, by incorporating the model into a game-theoretic structure with both imperfect and incomplete information. 
(1) Salop and Stiglitz [1987] also introduce product heterogeneity; the equilibrium they derive, however, does not feature any price dispersion.

(2) It is well known that mixed strategy equilibria of a game can be interpreted and justified as limits of pure strategy equilibria of the same game perturbed by an infinitesimal amount of incomplete information over players' types ; this, however, amounts to appealing to their heterogeneity. (3) While revising this paper, I became aware of Mc Minn's [ 1980] paper which already considered a particular model with both firm and consumer heterogeneity, corresponding to the assumptions of in Section II.1. The Iink between both sets of results is discussed in that section.

(4) For proofs, see Kohn and Shavel1 [1974] or Lippman and Mc Call [1976]. The latter also provides a discussion of the complications which arise when (a) or (b) fails; in particular, the optimal strategy with recall under riskaversion may not be a reservation price rule any more.

(5) If consumers have a finite maximum valuation $Z$ for the good, it will be assumed to be large enough not to constrain the equilibrium $(Z>\bar{r})$.

(6) Firms with $c=\bar{r}$ only matter when $\bar{r}$ coincides with an atom of $G$. Allowing for this occurrence, the most general form of (7) is :

$G\left(\bar{r}^{-}\right)<1 / 8<G\left(\bar{r}^{+}\right)=G(\bar{r})$. An equilibrium where double equality does not hold is unstable : a slight change in the distributions will alter $\bar{r}$ and it will not correspond to an atom any more. Also, any group of firms with $c=\bar{r}$ could decide to exit (say), thereby significantly altering the whole equilibrium. 
(7) When $\bar{r}=+\infty$, the bracketed term has a finite limit as $p \rightarrow+\infty$, hence $C_{F}(+\infty)=+\infty$. When $\bar{r}\langle+\infty(\Gamma(+\infty)\rangle \bar{\sigma})$, it is no smaller than $(q \circ \Gamma)(p) x(p) /$ $\int_{p}^{r}(q \circ \Gamma)(r) \times(r) d r$; if $q\left(\bar{\sigma}^{-}\right)>0$ this tends to to as $p$ tends to $\bar{r}$. If $q\left(\bar{o}^{-}\right)=0$, the minor additional assumption that $q$ is locally non increasing to the left of $\bar{\sigma}$ ensures that this term is larger than $1 /(\bar{r}-p)$, hence the result. (8) Under the standard assumption that $x(p)$ generates a we 11-behaved monopoly problem, i.e. that $p\left(1-1 / e_{x}(p)\right)$ is increasing (e.g. $x(p)$ concave). (9) In all generality, $\phi_{F}(p)=\theta\left(G \circ C_{F}\right)(p)$ for $p<\bar{r}_{F}$, where $1 / \theta$ is any number satisfying the inequality of footnote $(6) ; \phi_{F}$ is discontinuous at $\bar{r}_{F}$, and the equilibrium unstable, if equation (7) does not hold.

(10) It does when $x(p)=1$ (below some $Z>0$ ) and $q$ is increasing. Existence could probably be established in this case by showing that maps the space of c.d.f.'s on $[0, Z]$ into itself, and is continuous for the weak topology. Since the slightly stronger assumptions of Sections II and III allow a full resolution of the problem, this exercise does not seem worthwile. (11) Only upward discontinuities of $q$ on $[\sigma, \bar{\sigma})$ are consistent with the existence of a well behaved equilibrium.

(12) This is required to prevent all firms from charging the maximum price $Z$. (13) One thus leaves aside many Diamond-type equilibria with bunching, where all firms charge the same price $p \in[\bar{c}+\bar{\sigma}, Z]$, so that $p(c)=\underline{r}=p>(c+p+\bar{\sigma}) / 2=$ $(c+\bar{r}) / 2$ for all $c<\bar{c}$.

(14) Again, allowing for the coincidence of $\bar{r}$ with a discontinuity of $G$ : $(H / G)\left(\bar{r}^{-}\right) \geqslant 2 \bar{\sigma} \geqslant(H / G)(\bar{r})$. The stability reasoning of paragraph $I I .1 .1$ below can also be used to eliminate any solution without double equality. 
(15) This is required for the integrals defining reservation prices (e.g. $\left.\int_{0}^{r} p^{-\alpha} G(p(1-1 / \alpha)) d p\right)$ to be convergent in the neighborhood of $r=0$. Otherwise all consumers will search until they find the lowest price in the market, and the only equilibrium will be the competitive one, with only the most efficient $\operatorname{firm}(s)(c=c)$ operating. The same caveat applies to Reinganum $[19791$.

(16) Note from (20) that $C(r)>\underline{r}(1-1 / \alpha) \geqslant \underline{p}(1-1 / \alpha)=\underline{c}$, so that there is always bunching, contrary to case I.1.

(17) An alternative assumption would be that only the last firm where the good was bought can be revisited costlessly; however, it is hard to find an interpretation for such search costs (neither transportation nor inspection costs will do), when consumers have perfect memory.

(18) Since other histories can never be reached by a single player's deviation, the specification of equilibrium play after such a history leaves the equilibrium path unchanged.

(19) If consumers had a finite life, their reservation prices (for a constant price distribution) would increase with age, giving firms an incentive to conceal their cost type, then exploit loyal (old) consumers by raising prices, as in Bagwe 11 [1985]. An equilibrium might then involve price cycles. (20) As an additional justification, assume that returning to any $f \in I_{t, n}^{0}$, entails a small cost $\varepsilon>0$. The strategies and beliefs of Theorem IV.2 then constitute a (Bayesian perfect) $\varepsilon$-equilibrium, in which not returning to more than one $f \in K_{t, n}^{\sigma}$ at a time is not a w.d.s. any more, but in which returning to $f$ instead of searching may be suboptimal by max<0, $\varepsilon-\left[V\left(P_{t, n}^{\sigma}(f)\right)-V\left(R_{F}^{\sigma}(\sigma)\right)\right] /$ $(1-\delta)) \leqslant \varepsilon$. As $\varepsilon \rightarrow 0$, the search technology approaches the limiting case assumed in the Theorem, and the $\varepsilon$-equilibrium becomes a BPE. 
(21) Formula (34) is written for the case where the game has been played since $-\infty$. When it starts at some initial date $0,(34)$ still holds provided one defines $p_{k} \equiv p_{0}^{f}$ for $k<0$.

(22) The same remarks about consumers' return strategy as those which followed Theorem IV.2 still apply, however. 


\section{REFERENCES}

Axe 11, B. [1977] "Search Market Equilibrium", Scandinavian Journal of Economics, $79: 20-40$.

Bagwe 11, K. [1985] "Introductory Price as a Signal of Cost in a Model of Repeat Business", Review of Economic Studies, Vol. 54, $N^{\circ} 179: 365-384$.

Bénabou, R. [1986a] "Optimal Price Dynamics, Speculation and Search under Inflation", Ph.D. Dissertation, MIT.

[1986b] "Search, Price Setting and Inf lation", CEPREMAP Working Paper $n^{\bullet} 8622$; Review of Economic Studies, forthcoming.

[1987] "We Ifare Effects of Inf lation-Generated Search and Price

Dispersion" ; mimeo, CEPREMAP, June.

Diamond, P. [1971] "A Model of Price Adjustment", Journal of Economic

Theory, $3: 156-168$.

[1987] "Consumer Differences and Prices in a Search Model", Quarterly Journal of Economics, Vol. 12, $N^{\bullet} 2: 429-436$.

Fudemberg, D. and Tirole J. [1987] "Noncooperative Game Theory for Industrial Organization : An Introduction and Overview", MIT Working Paper, $N^{*}$ 445, April.

[1988] "Perfect Bayesian and Sequential Equilibrium:

A Clarifying Note" ; MIT, Working Paper, $N^{\bullet} 496$, May.

Gul, F., Sonnenschein, H. and Wilson R. [1986] "Foundation of Dynamic Monopoly and the Coase Conjecture", Journal of Economic Theory, 39, $n^{\circ} 1$ : 155-190.

Harsanyi, J. [1968] "Games with Incomplete Information Played by Bayesian Players 11 : Bayesian Equilibrium Points", Management Science, 14 : 320-334.

Kohn, M. and Shavell S. [1974] "The Theory of Search", Journal of Economic Theory, 9 : 93-123. 
Kreps, D. and Wilson, R. [1982] "Sequential Equilibria", Econometrica, 50 : 863-964.

Lippman, S. and McCa11, J. [1976] "The Economics of Job Search : A Survey", Economic Inquiry, 14 : 155-189.

Mc Millan, J. and Morgan, P. [1984] "Price Dispersion, Price Flexibility and Consumer Search", Working Paper, University of Western Ontario, August.

Mc Minn, R. [1980] "Search and Market Equilibrium", Journal of Political Economy, Vol. $88, N^{\bullet} 2: 308-327$.

Reinganum, J. [1979] "A Simple Model of Equilibrium Price Dispersion", Journal of Political Economy, 87 : 851-858.

Rob, R. [1985] "Equilibrium Price Distributions", Review of Economic Studies, 52 : 487-504.

Sa lop, S. and Stiglitz, J. [1987] "Information, Welfare, and Product Diversity", in "Arrow and the Foundations of the Theory of Economic Policy", edited by G.R. Feiwel. London, Macmillan.

Stiglitz, J. [1987] "Competition and the Number of Firms in a Market : Are Duopolies More Competitive Than Atomistic Markets ?" Journal of Political Economy, Vol. 95, $N^{\bullet} 5$ : 1041-1061.

Von zur Muehlen, P. [1980] "Monopolistic Competition and Sequential Search", Journal of Economic Dynamics and Contro1, 2 : 257-281. 


\section{APPENDIX}

\section{Proof of Theorem I :}

The subscript $F$ will be dropped from $C_{F}$ and $r_{F} ; d G$ (resp. dF) will denote the measure on the set of firms (resp. prices) defined by G (resp. F).

A - Sufficiency of the theorems' conditions : Let $F: R_{+} \rightarrow R_{+}$satisfy

$(a)-(b)$. By construction, $F(p)=b_{F}(p)$ tends to 1 as $p$ tends to $+\infty$, while $C(0)<0$, so that $F(0)=0 ; F$ is thus a c.d.f. on $R_{+}$. For all c $\in(c, \bar{r})$, $C$ has left and right limits $C\left(p^{-}\right)$and $c\left(p^{+}\right)=C(p)$ at $c$; define therefore :

$$
P(c)=\left\langle p \in[0, \bar{r}) \mid C\left(p^{-}\right) \leqslant c \leqslant C(p)\right\rangle
$$

Any local maximum of $\pi(. \mid c)$ must belong to $P(c)$, which generalizes the first order condition in (8), and is non-empty because $c(0) \leqslant 0<c$ and $c\left(\bar{r}^{-}\right)=\bar{r} \geqslant c$.

Lemma $A 1: P(c)$ is a singleton, for $d G$-almost all c .

Proof : For all $\left(p_{1}, p_{2}\right) \in[0, r)^{2}$, since both $G$ and $F=G o C$ are non-decreasing :

(A) $\left(p_{1}<p_{2}\right.$ and $\left.C\left(p_{1}\right)>C\left(p_{2}\right)\right) \Rightarrow G$ is constant on $\left[C\left(p_{2}\right), C\left(p_{1}\right)\right]$

Since $d G$ has no atom, this means that $\left[C\left(p_{2}\right), C\left(p_{1}\right)\right]$ has measure zero under dG. Consider now any $c \in(\underline{c}, \bar{r})$ and $\left(p_{1}, p_{2}\right) \in P(c)^{2}$, with $p_{1}<p_{2}$.

a) If $C\left(p_{1}\right)>C\left(p_{2}\right)$, then by (A1) $\left[C\left(p_{3}\right), C\left(p_{1}\right)\right]$ with $p_{3}=p_{2}-E$ and $E$ small enough has measure zero for $d G$. Thus $\left[C\left(p_{2}^{-}\right), C\left(p_{1}\right)\right]$, which contains c (since $\left.\left(p_{1}, p_{2}\right) \in P(c)^{2}\right)$, has measure zero for $d G$.

b) If $c\left(p_{1}\right)<c\left(p_{2}\right)$, then $c \leqslant c\left(p_{1}\right)<c\left(p_{2}\right) \leqslant c$ since $\left(p_{1}, p_{2}\right) \in P(c)^{2}$; thus $C\left(p_{1}\right)=C\left(p_{2}^{-}\right)$. Suppose that $C$ is not identically equal to $c$ on $\left[p_{1}, p_{2}\right)$, i.e. that there exists $p_{3}$ in this interval such that either: 
b1) $C\left(p_{3}\right)>c$; then $\left[c, C\left(p_{3}\right)\right]$ has measure zero for $d G$.

b2) $C\left(p_{3}\right)<c$; then $\left[C\left(p_{3}\right), c\right]$ has measure zero for $d G$.

Summing up cases (a) and (b) establishes that, except for a dG-negligible set of firm costs $c, C$ is constant on all $\left[p_{1}, p_{2}\right)$ where $\left(p_{1}, p_{2}\right) \in P(c)^{2}$. In other words, for dG-almost all $c:(i) P(c)$ is an interval $\left[p^{-}(c), p^{+}(c)\right]$ (or $\left[p^{-}(c), p^{+}(c)\right)$ if $c$ is discontinuous at $\left.p^{+}(c)\right)$, and if this interval has positive length, then $C$ is constant and equal to $c$ on it; (ii) for $p<p^{-}(c), C(p)<c ;(i i i)$ for $p>p^{+}(c), C(p)>c$ (for dG-almost all c).

Consider now any interval $I$ on which $G$ is increasing, f.e. which does not contain any subinterval of measure zero under $d G$. Define, for all c $E I$, the function $p(c) \equiv p^{+}(c)$. It is non-decreasing, because $c_{1}>c_{2}$ and $p^{+}\left(c_{1}\right)<p^{+}\left(c_{2}\right)$ implies $c\left(p^{+}\left(c_{1}\right)\right)<c_{2}<c_{1}<c\left(p^{+}\left(c_{2}\right)\right)$, (by (i) to (iii) above) and it was seen that this requires $\left[C\left(p^{+}\left(c_{1}\right)\right), C\left(p^{+}\left(c_{2}\right)\right)\right]$ and its subinterval $\left[c_{2}, c_{1}\right]$ to have measure zero for $d G$, a contradiction. As a nondecreasing function, $p(c)$ has at most a countable number of discontinuities ; these discontinuities are easily seen to correspond to points c such that : $\bar{p}(c)<p^{+}(c)$, so the set of $c \in I$ such that $P(c)$ has more than one element is countable. Since this is true for every interval I which contains a nonnegligible mass of firm costs, and since there are no atoms in the cost distribution ( $G$ is continuous), the set of $c \in[C, \bar{r}]$ such that $P(c)$ is not a singleton has measure zero under $G$; this concludes the proof of Lemma $A 1$. We now show that $F$ is an equilibrium price distribution.

Let firms with $c>\bar{r}$ not operate. For all $c<\bar{r}, \pi(. \mid c)$ has a global maximum on $[c, \bar{r}]$, which must also be a local maximum (since $\pi(c \mid c)=\pi(\bar{r} \mid c)=0$ ), hence 
an element of $P(c)$. But, for $d G$ almost all firms, $P(c)$ is a singleton $(p(c))$ (with $C(p(c))=c)$, implying so that $p(c)$ is indeed a globally optimal price for a firm with cost c. Moreover, for any such firm :

* if $p \geqslant p(c)=p^{+}(c)$, then $C(p) \geqslant c$ by $(i)$ and $(i i i)$ above;

* if $p<p(c)=p^{-}(c)$, then $C(p)<c$ by $(i i)$ above ;

so that $p(c)<p$ if and only if $C(p)<p$, for dG-almost all $c$. The number of prices no greater than $p$ charged in the market is therefore $G(C(p))$, which by hypothesis is $F(p) G(\bar{r})$. Hence : $\operatorname{Prob}(p(c)<p)=F(p)$.

B - Necessity of the theorem's conditions : Let $F$ be an equilibrium such that the set of firms with more than one local maximum in their profit function $\pi(. \mid c)$ has measure zero under $d G$.

Lemma $A 2$ : For all $\left(p_{1}, p_{2}\right) \in[0, \bar{r}]^{2}$ such that $p_{1}<p_{2}$ and $C\left(p_{1}\right)>C\left(p_{2}\right)$, the interval $\left[C\left(p_{2}\right), C\left(p_{1}\right)\right]$ has measure zero under $d G$.

Proof : Since $G$ has no atoms and its support is contained in $[0,+\infty)$, the proposition is immediate when $C\left(p_{1}\right)=C\left(p_{2}\right)$ or $c\left(p_{1}\right)<0$. Assume from now on that $\left(C\left(p_{2}\right), C\left(p_{1}\right)\right) \cap R_{+}^{*} \neq \varnothing$ and consider any $c$ in this interval.

Denote by $\pi_{d}^{\prime}(p \mid c)=-D^{\prime}(p)(c-c(p))$ the right derivative of $\pi(. \mid c)$ at every point ; then $c(0)<0<c<c\left(p_{1}\right)$ implies that $\pi_{d}^{\prime}(0 \mid c)>0>\pi_{d}^{\prime}\left(p_{1} \mid c\right)$. The continuous function $\pi(. \mid c)$ restricted to $\left[0, p_{1}\right]$, has a global maximum ; since $\pi_{d}^{\prime}(0 \mid c)>0$, it cannot occur at zero, so two cases are possible :

a) $\pi(. \mid c)$ possesses an interior, and therefore local, maximum in $\left(0, p_{1}\right)$.

b) $\left(\forall p \in\left(0, p_{1}\right)\right)\left(\pi(p \mid c)<\pi\left(p_{1} \mid c\right)\right)$. But since $\pi_{d}^{\prime}\left(p_{1} \mid c\right)<0$ :

$(\exists \varepsilon>0)\left(V p \in\left[p_{1}, p_{1}+\varepsilon\right), \pi(p \mid c)<\pi\left(p_{1} \mid c\right)\right)$

hence $p_{1}$ is a local maximum of $\pi(. \mid c)$. 
Thus in both cases, $\pi(. \mid c)$ possesses a local maximum in $\left[0, p_{1}\right]$. Similarly, using the fact that $c\left(p_{2}\right)<c<c\left(p_{1}\right)<p_{1}<\bar{r}=c\left(\bar{r}^{-}\right)$, one shows that $\pi(. \mid c)$ has a local maximum in $\left(p_{2}, \bar{r}\right)$. Thus, for all $c \in\left(c\left(p_{2}\right), c\left(p_{1}\right)\right) \cap R_{+}^{*}, \pi(. \mid c)$ has more than one local maximum, which (by assumption) implies that $\left(C\left(p_{2}\right)\right.$, $\left.C\left(p_{1}\right)\right)$ has measure zero under $d G$. Since $G$ has no atoms, the same holds for $\left[C\left(p_{2}\right), C\left(p_{1}\right)\right]$, which concludes the proof of the lemma.

The number of firms charging a price $p(c)<p$ in equilibrium will now be computed. For any $c \in[\underline{c}, \bar{r})$, consider the following cases, for any given $p$ :

a) If $c<C(p)$ and $p(c)>p$; since any optimal price $p(c)$ must belong to $P(c), C\left(p(c)^{-}\right)<c$ so: $p_{1} \equiv p<p(c)-E \equiv p_{2}$ and $c\left(p_{1}\right)>C\left(p_{2}\right)$ for all $E$ small enough. By Lemma $A 2,\left[C\left(p_{2}\right), C\left(p_{1}\right)\right]$ has measure zero; thus $[C, C(p)]$ has measure zero under $d G$.

b) If $c>c(p)$ and $p(c) \leqslant p$; since $c(p(c)) \geqslant c$ because $p(c) \in P(c)$, $p_{1} \equiv p(c)\left\langle p \equiv p_{2}\right.$ and $C\left(p_{1}\right)>c>C\left(p_{2}\right)$, which requires $[C(p), c]$ to have measure zero under $d G$.

Thus, if $c_{1}$ and $c_{2}\left(c_{1}<c_{2}\right)$ satisfy $(b)$ (remember that $p$ is fixed), then $\left[c_{1}, c_{2}\right]$, as a subset of $\left[C(p), c_{2}\right]$, has measure zero under $d G$. Costs satisfying (b) are therefore separated by intervals of measure zero under $d G$; as $d G$ has no atoms, the set of such costs itself must also have measure zero. Since the same applies to costs satisfying (a), the following property holds for $d G$-almost all costs $c$ :

$$
(p(c)<p \Leftrightarrow c<c(p))
$$


The "number" of firms charging a price no greater than a given $p$ is therefore $G(C(p))$; normalizing by $1 / \theta=G(\bar{r})$ yields $F(p)={ }_{F}(p)$ for all $p$. Q.E.D.

Proof of Theorem II.I:

Only existence remains to be established. The function $H / G$ has left and $r$ ight limits everywhere on $(c,+\infty)$ and is right-continuous, because $G$ has these properties and $H$ is continuous. For $r \geqslant \bar{c},(H / G)(r)=\int_{0}^{\bar{c}} G(c) d c+r-\bar{c}$, hence $(H / G)(+\infty)=+\infty$. Moreover, for allc $<r, G(c)<G(r)$ so: $(H / G)(r)<\int_{c}^{r} d c=r-\underline{c}$, hence $(H / G)\left(c^{+}\right)=0$. Therefore for a $11 \bar{\sigma}>0$, there exists $r>0$ such that : $(H / G)\left(r^{-}\right)<2 \bar{\sigma}<(H / G)\left(r^{+}\right)=(H / G)(r)$. But any discontinuity in $G$ is upward, so any discontinuity in $H / G$ is downward. The preceding double inequality can therefore only be a double equality (with intersection from below). Q.E.D.

Proof of Theorem 1I.2 :

It remains to be shown that if $g / G \equiv G^{\prime} / G$ is decreasing on $I=[c,+\infty)$, so is $H^{\prime} / H=G / H$. Since $H / G$ is continuous on $I$, with limits of 0 and $+\infty$ at $c^{+}$and $+\infty$ respectively (cf. proof of Theorem II.1.1), it is increasing if and only if : (A3)

$$
(\forall \lambda>0)\left(\exists ! c_{\lambda}>c\right)\left(H\left(r_{\lambda}\right) / G\left(r_{\lambda}\right)=\lambda\right)
$$

Define, for all $\lambda>0$, the function: $J_{\lambda}(r)=H(r)-\lambda G(r)$ for c $E[\underline{c},+\infty)$. Then (A3) is equivalent to :

$$
(\forall \lambda>0)\left(3 ! r_{\lambda}>0\right)\left(J_{\lambda}\left(r_{\lambda}\right)=0\right) .
$$

The function $J_{\lambda}$ is continuously differentiable on $I$, and (A5)

$$
(\forall r \in I) \underset{\lambda}{\left(J^{\prime}(r)=G(r)[1-\lambda(g / G)(r)]\right) .}
$$

But $G$ is non-decreasing and positive on $I$, while $g / G$ is decreasing by 
assumption ; thus by (A5) there exists a unique $c_{\lambda} \geqslant \underline{c}$, such that $J$ is decreasing on $\left[\underline{c}, c_{\lambda}\right]$ (which is possibly empty) and increasing (to a limit of $+\infty$ ) on $\left[c_{\lambda},+\infty\right)$. Since $J_{\lambda}(c)=-\lambda G(c)<0, J_{\lambda}$ has no positive root in. $\left[c_{-}, c_{\lambda}\right]$. On $\left[c_{\lambda},+\infty\right)$, on the contrary, $J_{\lambda}$ increases from $J_{\lambda}\left(c_{\lambda}\right)<0$ to a limit of +o. Hence (A4) holds.

Proof of Lemma 1 :

Let $k \equiv \beta-r ;\left(C^{*}\right)^{\prime}(p)$ has the sign of :

$$
\left(2+k p^{\alpha-1}\right)\left(2 \alpha-1+\alpha k p^{\alpha-1}\right)-(\alpha-1) k p^{\alpha-1}=\alpha\left(2+k p^{\alpha-1}\right)\left(1+k p^{\alpha-1}\right)+2(\alpha-1)
$$

which is clearly positive when $k>0$. Since $\beta / \bar{r}=0$ and $\alpha>1$, the case $k<0$ requires $\beta=0, k=-r^{1-\alpha}$ so $k p^{\alpha-1}>-1$ hence again $\left(C^{*}\right)^{\prime}(p)>0$ Q Q.E.D.

\section{Proof of Theorem II.3:}

Only existence remains to be shown ; let $\mu=1-1 / \alpha$.

Case 1: $\int_{0}^{\infty} p^{-\alpha} G(p(1-1 / \alpha)) d p<\underline{0}$.

Let all firms charge $p^{m}(c)=c / \mu$. Then $F(p)=G(\mu p)$ is an equilibrium distribution, because $\Gamma_{F}(+\infty)<\underline{a}$, or $r_{F}=+\infty$, so that no consumer searches : thus $(r, \bar{r}, \beta)=\left(+\infty,+\infty,(\alpha-1)\left[\bar{\sigma}-\int_{0}^{\infty} p^{-\alpha} G(\mu p) d p\right]\right)$ solves $(24 a)$ to $(24 c)$.

Case $2: \int_{0}^{\infty} p^{-\alpha} G(p(1-1 / \alpha)) d p>\underline{0}$.

The function $T_{0}:\left[u \rightarrow \int_{0}^{u} p^{-\alpha} G(\mu p) d p\right]$ is continuous and increasing on $[\underline{c} / \mu,+\infty)$, from 0 at $\underline{c}>0$ to a limit $\mathrm{T}_{0}(+\infty)>\underline{c}$. Therefore :

(A6) $(\forall r \geqslant c)(\exists ! U(r) \geqslant c / \mu)\left(\int_{0}^{u(r)} p^{-\alpha} G(\mu p) d p=G(r) \underline{a}\right)$ By $(A 6), u($.$) is non-decreasing and right-continuous. Note that, for r$ large enough, $G(r)$ is arbitrarily close to 1 , hence $u(r)$ close to the finite solution 
of $T_{0}(u)=\underline{a}$, so $r>u(r)$. Define now for all $(w, r)$ with $r \geqslant w \geqslant \underline{c}$ :

$$
T(w \mid r, 0)=\underline{\sigma}+\int_{u(r)}^{w} p^{-\alpha}\left[G\left(C^{*}(p \mid r, 0)\right) / G(r)\right] d p
$$

with $C^{\star}(p \mid \bar{r}, \beta)$ defined by $(20)$ and $\beta=0$ here. This function is continuous in $w$ and right-continuous in $r$, with left and right limits in $r$ at every point. Let us first concentrate on the case where $w=r$, i.e. on the function $T(r) \equiv T(r \mid r, 0)$. When $r$ is close to $\underline{c}^{+}, u(r)>\underline{c} / \mu>r$ so:

(AB) $\quad T(r)=\underline{\sigma}+\int_{U(r)}^{r} p^{-\alpha}\left[G\left(C^{*}(p \mid r, 0)\right) / G(r)\right] d p<\underline{\sigma}<\bar{\sigma}$

and two cases arise.

Case 2.1 $:(\exists r \in(\underline{c},+\infty))\left(T\left(\bar{r}^{-}\right)<\bar{\sigma}<T(\bar{r})\right)$.

But $G\left(\bar{r}^{-}\right)<G(\bar{r})$ and $u\left(\bar{r}^{-}\right)<u(\bar{r})$ imply by $(A 8), T\left(\bar{r}^{-}\right) \geqslant T(\bar{r})$, so the above must be a double equality, with the intersection between $T(r)$ and the horizontal $\bar{\sigma}$ occuring from below (which is easily seen, as in Section 11.1 .1 , to correspond to stability). Assume now that firms behave as claimed in the theorem, with the above defined $\bar{r}, \underline{r} \equiv u(\bar{r})$ and $\beta=0$; the claimed form (22) of $F(p)$ follows immediately (note from (A5) that it is indeed increasing on $[c / \mu,+\infty])$, as well as consumers' optimal strategies from the general formula (3). Thus, from the above definition of $\bar{r},(22),(A 6)$ and $(A 7), r_{F}(r)$ is equal to :

$$
\left\{\begin{array}{l}
(\forall r<\underline{r})\left(\Gamma_{F}(r)=T_{0}(r) / G(\bar{r})\right) \\
(\forall r \in[\underline{r}, \bar{r}])\left(\Gamma_{F}(r)=T(r \mid \bar{r}, 0)\right)
\end{array}\right.
$$

$\left(A 9^{\prime}\right)$

$$
r_{F}(r)=T_{0}(u(\bar{r})) / G(\bar{r})=0 ; r_{F}(\bar{r})=T(\bar{r} \mid \bar{r}, 0)=T(\bar{r})=\overline{0} .
$$


Since $r_{F}$ is increasing on $[c / \mu,+\infty),\left(A 9^{\prime}\right)$ implies that $(r, \bar{r}, 0)$ indeed solves the equilibrium equations $(24 a)-(24 c)$.

Case $2.2:(\forall r>c)(T(r)<\bar{\sigma})$.

The fixed-point requirement will bear in this case on $\beta$ rather than $\bar{r}$. Define $\bar{r}=+\infty$ and $\underline{r}=u(+\infty)$, i.e. the only solution to :

$$
\int_{0}^{r} p^{-\alpha} G(\mu p) d p=G(+\infty) \cdot \underline{0}=\underline{0}
$$

As $r$ tends to $+\infty, u(r)$ tends to $r$ and, since the integrand in (AB) is positive, $T(r)$ tends to a limit $T(+\infty)$, with $T(+\infty) \leqslant \bar{\sigma}$ by assumption. For all $\beta \geqslant 0$, define now the following functions on $[\underline{c},+\infty]$ :

(A11)

$$
\begin{cases}T(w \mid+\infty, \beta)=T_{0}(w)=\int_{0}^{w} p^{-\alpha} G(\mu p) d p & \text { for } w<\underline{r} \\ T(w \mid+\infty, \beta)=\underline{a}+\int_{r}^{w} p^{-\alpha} G\left(C^{*}(p \mid+\infty, \beta)\right) d p \text { for } w \geqslant \underline{r}\end{cases}
$$

Note from (A4) that $C^{\star}(p \mid+\infty, \beta)$ is continuous and decreasing in $\beta ;(A 11)-(A \mid 2)$ then imply that $L$ is right-continuous in $\beta$, and can only have downward discontinuities. Since $L(+\infty)=+\infty$ and :

$$
L(0)=T(+\infty \mid+\infty, 0)=\underline{\sigma}+\int_{r}^{\infty} p^{-\alpha} G\left(C^{*}(p(+\infty, 0))=T(+\infty)<\bar{\sigma},\right.
$$

there exists $\beta * \in[0,+\infty)$ with $L\left(\beta_{*}\right)=\bar{\sigma}$ (and intersection from below), i.e.:

$$
\beta x=(\alpha-1)(\bar{\sigma}-T(+\infty \mid+\infty, \beta x))
$$

This is nothing else than equation $(24 c)$, for $(r, \bar{r}, \beta)=(u(+\infty),+\infty, \beta *)$. Moreover, from the definition of $F$ in $(22)$, and from $(A 10)$ and $(A 11), r_{F}(r)$ is equal to $T(r \mid+\infty, \beta *)$, for all $r$. In particular, by $(A 10)$ and $(A 13)$ : 


$$
\Gamma_{F}(r)=T(r \mid+\infty, \beta *)=T_{0}(r)=\underline{\sigma} ; \Gamma(\bar{r})=T(+\infty \mid+\infty, \beta *)=\bar{\sigma}-\beta * /(\alpha-1) \leqslant \sigma
$$

so that $(24 a)$ and $(24 b)$ are satisfied as well by $(u(+\infty),+\infty, \beta *)$. Q.E.D.

Proof of Theorem II.4:

We examine the same cases as in the proof of Theorem 11.2.1, imposing the additional constraint $G(\bar{r})=1$. Case 1 , where $r=\bar{r}=+\infty$ remains unchanged. In Case $2, r$ is now simply determined as the unique solution to :

$$
\int_{0}^{r} p^{-\alpha} G(\mu p) d p=0
$$

The rest of the analysis (existence of $\bar{r}$ and $\beta$ ) then goes through as before, with $G(\bar{r})$ replaced by 1 everywhere ; it remains to be checked, however, that any solution $\bar{r}$ thus obtained satisfies $\bar{r} \geqslant \bar{c}$. Under $(i)$, the above equation implies $\underline{r} \geqslant \bar{c}$, hence $\bar{r} \geqslant \bar{r} \geqslant \bar{c}$. If (i) does not hold but (ii) does, then $\underline{r} \in(\underline{c}, \bar{c})$ but, for all $r \in[\underline{r}, \bar{c})$ :

$$
\int_{\underline{r}}^{r} p^{-\alpha} G\left(C^{\star}(p \mid \bar{r}, 0)\right) d p<\int_{c}^{r} p^{-\alpha} G(p) d p<\int_{\underline{c}}^{\bar{c}} p^{-\alpha} G(p) d p<\bar{\sigma}-\underline{a}
$$

since $C^{*}(p \mid \bar{r}, 0)<p$ for all $p<\bar{r}$. Thus $T(r)<\bar{\sigma}$ on $[r, \bar{c})$ so that $\bar{r}$, which is either a solution to $T(r)=\bar{\sigma}($ case 2.1$)$ or $\bar{r}=+\infty($ case 2.2$)$ if no such solution exists, must be no smaller than $\bar{c}$. Q.E.D. Proof of Theorem IV.2 :

A- Consumer strategies : Firms' strategies (32) prescribe prices $\tilde{p}\left(H_{t}^{f}\right)$ which - possibly after a single adjustment - remain constant over time, whatever prices were charged in the past, and which - excluding simultaneous deviations of positive measure - induce the price distribution $F(p)$. At any information set, consumer o thus faces (or at least, believes with probability 
one he is facing) a distribution of constant price paths. Using this observation, we now characterize his dynamic programming problem. For any history $H$ of consumer $\sigma$, let $I(H)$ denote here the set all previously visited firms, $P(f \mid H)$ the highest price ever observed at any $f \in I(H), W(H)$ the value of an optimal program when o starts from "home" (i.e. is not visiting a seller) with information set $H$, and $W(H ; p)$ the value of an optimal program when 0 starts from a firm $f \in I(H)$ charging a price $p=P(f \mid H)$ which is expected to remain constant forever. In this last situation, o can either buy from the firm or leave, and in both cases his relevant information remains unchanged, so that we can write :

$$
\text { (A14) } \tilde{W}(H ; P)=\max (V(p)+\delta W(H), W(H))
$$

When $\sigma$ is "at home", or between firms, he can either go to some $f \in I(H)$, which by assumption he expects (with probability one) to charge $P(f \mid H)$, or sample a firm at random. In the first case he does not expect to gain any new information, while in the second he will discover a new firm f' charging a price $p^{\prime}$ (which he believes to be permanent; his new information set will then be denoted (for brevity) by $H^{\prime}=H U\left\langle p^{\circ}\right\rangle$. Thus :

$$
\text { (A15) } W(H)=\max \left\{\max _{f \in I(H)} \tilde{W}(H ; P(f \mid H)),-0+\int_{-\infty}^{+\infty} \tilde{W}\left(H \cup\left(p^{\prime}\right) ; p^{\prime}\right) d F\left(p^{\prime}\right)\right\}
$$

where the maximum over $f \in I(H)$ is taken to be $-\infty$ when this set is empty. The Bellman equation obtained by substituting (A14) into (A15) defines a's dynamic programming problem at $H$. It is easily verified (by substituting them in, and using the definition $(31)$ of $R_{F}^{\delta}(a)$ ) that the functions : 
(A16)

$$
W(H)=\tilde{W}(H, p)=\frac{1}{1-\delta} \max \left\{\max _{f \in I(H)} V(P(f \mid H)), V\left(R_{F}^{\delta}(\sigma)\right)\right\}
$$

$(\tilde{W}(H ; p)$ is defined for all $p$ such that there exists $f \in I(H)$ with $p=P(f \mid H))$ are solutions to $(A \mid 4)-(A 15)$. Moreover, this expected value is clearly reached by the algorithm described in Theorem IV.2, since, in particular, searching at random until a price $p<R_{F}^{\delta}(\sigma)$ is found and then purchasing repeatedly at this price yields $V\left(R_{F}^{\delta}(\sigma)\right) /(1-\delta)$. This algorithm therefore defines an optimal solution, for any consumer history $H$.

B - Firms' strategies : Simultaneous deviations by consumers are excluded. Therefore, for any price path $\left\langle p_{0}, \ldots, p_{t-1}\right\}$, followed by a firm $f$ (the superscript $f$ will be omitted for simplicity) only the consumers who encountered it and have reservation prices above $P_{t-1} \equiv \max _{s}|s<t-1\rangle$ remain at time $t$, and all of them remain. Since other firms do not deviate, no new consumers have arrived or can be expected to arrive in the future. As of period $t$, the distribution of search reservation prices among the firm's clientele is therefore the initial one, given by (4), except that it is truncated below $P_{t-1}$. Since this also holds for any $s \geqslant t$, the demand curve after any firm history $H_{s}^{f}$ is $D_{s}^{f}(p)=D\left(\max \left\langle p, P_{s-1}\right\}\right)$, where $D$ is given by $(5)$. The firm after $H_{t}^{f}$ thus chooses $\left\langle p_{s} \mid s \geqslant t\right\rangle$ to maximize :

$$
\begin{aligned}
\left.\pi\left(\left\langle p_{s}\right| s \neq t\right) \mid H_{t}^{f}\right) & =\varepsilon_{s=t}^{\infty} \delta \delta_{s}^{s-t}\left(p_{s}-c\right) D\left(\max \left(p_{s}, P_{s-1}\right)\right) \\
& =\sum_{s=t}^{\infty} \delta{ }^{s-t}\left(p_{s}-c\right) D\left(P_{s}\right)
\end{aligned}
$$

where $c=c^{f}$. For any $s \geqslant t$ :

$$
\left.\left(p_{s}-c\right) D(P)_{s}\right) \leqslant\left(P_{s}-c\right) D(P)_{s}<(p(c)-c) D(p(c))
$$


with double equality if and only if $p_{s}=P_{s}=p(c)$. So, if $P_{t-1}<p(c)$, the optimal strategy is to set $p_{s}=p(c)$, hence $P_{s}=p(c)$ for all future $s$. If $P_{t-1}>p(c)$, on the contrary, then for any sequence $\left.\left\langle p_{s} \mid s\right\rangle t\right\rangle$, $P_{s} \geqslant P_{t-1}>p(c)$. Since $(p-c) D(p)$ is decreasing on $(p(c),+\infty)$ :

$$
\left(p_{s}-c\right) D\left(P_{s}\right)<\left(P_{s}-c\right) D\left(P_{s}\right)<\left(P_{t-1}-c\right) D\left(P_{t-1}\right)
$$

for all $s \geqslant t$, with equality if and only if $P_{s}=P_{t-1}$. The optimal strategy is therefore to set $p_{s}=P_{s}=P_{t-1}$ for all s $\geqslant t$. Thus, for any history $H_{t}^{f}$ :

$$
\tilde{p}\left(H_{t}^{f}\right) \equiv \max \left(p(c), P_{t-1}\right)
$$

$c$ - Updating of beliefs: Along the equilibrium path, each firm $f$ charges $p_{F}^{\delta}\left(c^{f}\right)$ in every period; each consumer o initially searches according to

$R_{F}(\sigma(1-\delta))$ and then remains attached to his first supplier. Thus consumers' beliefs (with probability 1) that a firm's optimal static price $p_{F}^{\delta}(c)$ ) is the highest price observed there and that it is currently charging that same price do obey Bayes's rule for updating according to firms' strategies (and they are always true). The same holds for firms' beliefs that all customers from the previous period have decided to come back. Beliefs off the equilibrium path are unrestricted by Bayes' rule, but, even there, consumers' beliefs about the yet unobserved price of a previous supplier are consistent with their belief about its cost type and their knowledge of each type's strategy (A16). Q.E.D.

\section{Proof of Lemma 2 :}

Consumers' strategies and beliefs call for them to leave and never return whenever they observe a price greater than their reservation price. Consider a firm $f$ with cost $c^{f}=c$ at an information set $H_{s}^{f}$. Its clientele is composed of 
the sum, for all $\tau, 0 \leqslant \tau \leqslant s$, of those consumers who joined at date $s-r$, have "survived" since, and have a reservation price above the maximum price charged by the firm since that date: $P_{s-\tau, s}^{f} \equiv \max _{k} p_{k}^{f}|s-\tau \leqslant k \leqslant s\rangle$. In any generation, the demand per firm from consumers with $R_{F}^{\delta \mu}(\sigma) \geqslant P_{S-\tau, s}^{f}$ is $\theta \lambda D_{F}^{\delta \mu}(P, s, s)$, with $D_{F}^{\delta \mu}($.$) given by (5). For simplicity the indices f, \delta, \mu, F$ will be omitted from $c, P^{f}, \rho_{f}^{\delta \mu}, D_{F}^{\delta \mu}$, etc. Summing up over all such generations between 1 and $s$, and adding consumers from the initial (time zero) stock which has density $\lambda / \mu$ yields :

$$
\begin{aligned}
& D\left(p_{S} \mid H_{S}^{f}\right)=\theta \lambda\left[_{T=0}^{S-1}(1-\mu)^{\tau} D\left(P_{S-\tau, S}\right)+(\theta \lambda / \mu)(1-\mu)^{S} D\left(P{ }_{0, S}\right)\right. \\
& =\theta \lambda \sum_{\tau=0}^{S-1}(1-\mu)^{\tau} D\left(P S_{S-\tau, S}\right)+\theta \lambda \sum_{\tau=S}^{\infty}(1-\mu)^{T} D\left(P S_{S-\tau, S}\right) \\
& =\theta \lambda \sum_{\tau=0}^{\infty}(1-\mu)^{\top} D\left(P_{S-\tau, S}\right)
\end{aligned}
$$

with the convention that $P_{S-\tau, S}=P_{0, s}$ for all $\tau \geqslant s$ (equivalently, one could consider the market in operation since $t=-\infty$ ). Summing up the discounted profits corresponding to these demands, over $s \geqslant t$ yields (34). A solution to the infinite-horizon program of maximizing this expected present value of profits over $\left\langle p_{s} \mid s \geqslant t\right\rangle$ will now be constructed, as the limit, as $T$ goes to infinity, of the solutions to the finite horizon problems obtained by truncating the original one at $T>t$. Let thus $H_{t}^{f}$ be given; the dependence of $\pi$ on $H_{t}^{f}$ will now be dropped for notational simplicity. For any $T>t$, and any infinite sequence $\left\langle p_{S} \mid s \geqslant t\right\rangle$, denote by $\pi_{T}\left(\left\langle p_{s}\right\rangle\right)$ the firm's discounted profits from to $T$ on $1 y$. Since $\pi_{T}\left(\left\langle p_{s}\right\rangle\right)$ depends only on $\left\langle p_{s} \mid t \leqslant s \leqslant T\right\rangle$, it trivially defines a function (which will also be denoted as $\pi_{T}$ ) on $R_{+}^{T-t+1}$. The finite horizon problem consists in maximizing ${ }_{T}$ on that space. 
Claim 1 : In the finite horizon problem, charging $p<p(c)$ is never optimal.

Indeed, for any sequence $\left\{p_{s} \mid t \leqslant s \leqslant T\right\}$, defining :

$$
\begin{gathered}
\left(q_{s}=\max \left(p_{s, p}(c)\right) \mid t<s<T\right) \text {, one can write for } a l l s \geqslant t \text { and } \tau \geqslant 0: \\
\left.Q_{s-\tau, s} \equiv \max _{k}\left(q_{k} \mid s-\tau<k \leqslant s\right\}=\max _{s-\tau, s}, p(c)\right\}
\end{gathered}
$$

and consider the following two cases.

Case 1 : If $P_{s-\tau, s}<p(c)$; since $(p-c) D(p)$ is increasing on $[c, p(c)]$ :

$$
\begin{aligned}
\left(p_{s}-c\right) D\left(P_{s-\tau, s}\right) & <\left(P_{s-\tau, s}-c\right)\left(D\left(P_{s-\tau, s}\right)\right. \\
& <(p(c)-c) D(p(c))=\left(q_{s}-c\right) D\left(Q_{s-\tau, s}\right) .
\end{aligned}
$$

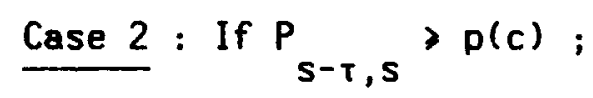

$$
\left(p_{s}-c\right) D\left(P_{s-\tau, s}\right)=\left(p_{s}-c\right) D\left(Q_{s-\tau, s}\right)<\left(q_{s}-c\right) D\left(Q_{s-\tau, s}\right)
$$

with strict inequality if $p_{s}<q_{s}$, i.e. $p_{s}<p(c)$. Thus in both cases :

$$
\begin{aligned}
& \pi_{T}\left(\left\langle p_{s}\right\rangle\right)=\theta \lambda \sum_{s=t}^{T} \delta \delta^{s-t} \sum_{\tau=0}^{\infty}(1-\mu)^{T}\left(p_{s}-c\right) D\left(P{ }_{s-\tau, s}\right) \\
& <\theta \lambda \sum_{s=t}^{T} \delta^{s-t} \sum_{T=0}^{\infty}(1-\mu)^{T}\left(q_{s}-c\right) D\left(Q_{s-\tau, s}\right) \text {, or : }
\end{aligned}
$$

$$
\pi_{T}\left(\left\langle p_{S}\right\}\right)<\left(\left(\max \left(p(c), p_{S}\right)\right)\right)
$$

with strict inequality if any $p$ is smaller than $p(c)$.

Claim 2 : In the finite horizon problem, $p>\max \left\{p(c), P_{t+1}\right.$ is never optimal. Assume first that $P_{t-1} \leqslant p(c)$; by $(A 17), p_{s}=p(c)$ for all $s \geqslant t$ maximizes $\pi_{T}$. Assume that on the contrary, $P_{t-1}>p(c) ;$ redef ine the sequences (q $q_{s}$ ) and $\left\langle Q_{s-\tau, s}\right.$ ) as : $\left.q_{s}=\min _{s}, p_{t-1}\right\rangle, Q_{s-\tau, s}=\max _{k}\left(q_{k} \mid s-\tau<k<s\right)$ $=\min \left\langle P\left(s-\tau, s, P_{t-1}\right\rangle\right.$. For any $s \geqslant t$ and $T \geqslant 0$, three cases are possible. 
Case 1 : If $P_{s-\tau, s} \leqslant P_{t-1}$; then $\left(p_{s}-c\right) D\left(P P_{s-\tau, s}\right)=\left(q_{s}-c\right) 0\left(Q_{s-\tau, s}\right)$.

Case 2 : If $P_{s-\tau, s}>P_{t-1}>p(c)$ and $p_{s} \geqslant P_{t-1}$; then $\left(p_{s}-c\right) D\left(P P_{s-\tau, s}\right)<$

$$
\left(P_{s-T, s}-c\right) D\left(P_{s-\tau, s}\right)<\left(P_{t-1}-c\right) D\left(P_{t-1}\right)=\left(q_{s}-c\right) D\left(Q_{s-T, s}\right) \text {. }
$$

Case 3 : If $\max \left(p(c), p_{s}\right)<P_{t-1}<P_{s-\tau, s}$; then $\left(p_{s}-c\right) D\left(P_{s-\tau, s}\right)<$

$$
\left(p_{s}-c\right) D\left(P_{t-1}\right)=\left(p_{s}-c\right) D\left(Q_{s-\tau, s}\right)=\left(q_{s}-c\right) D\left(Q_{s-\tau, s}\right) .
$$

Thus, in every case, $\left(p_{s}-c\right) D\left(P_{s-\tau, s}\right)<\left(q_{s}-c\right) D\left(Q_{s-\tau, s}\right)$, with strict inequality if and only if $P_{s-\tau, s}>P_{t-1}$. Hence :

$$
\begin{aligned}
& \pi_{T}\left(\left\langle p_{S}\right\rangle\right)=\theta \lambda \sum_{S=t}^{T} \delta \delta_{T=0}^{s-t} \sum_{T}^{\infty}(1-\mu)^{T}\left(p_{S}-c\right) D\left(P{ }_{S-\tau, S}\right) \\
& \text { \& } \theta \lambda \sum_{s=t}^{T} \delta^{s-t} \sum_{\tau=0}^{\infty}(1-\mu)^{\tau}\left(q_{s}-c\right) D\left(Q_{s-\tau, s}\right)
\end{aligned}
$$

with strict inequality if and only if there exists some $s \geqslant t$ and $t \geqslant 0$ such that $P_{s-\tau, s}>P_{t-1}$, i.e. there exists $k>t$ such that $p_{k}>P_{t-1}$. Thus :

(A20)

$$
\left.\pi_{T}\left(\left\langle p_{S}\right)\right)<\pi_{T}\left(\sin \left(p_{S}, P_{t-1}\right)\right)\right)
$$

with strict inequality if $p_{k}>P_{t-1}$ for some $k \geqslant t$.

On the compact $Q_{t, T} \equiv\left[p(c), \max \left(p(c), P_{t-1}\right)\right]^{T-t+1}$, the function $\pi_{T}$ is continuous, and therefore attains its maximum at some $\left\langle\mathrm{p}_{T, s} \mid t<s<T\right\rangle$, with : (A21)

$$
p(c) \leqslant p_{T, s} \leqslant \max \left(p(c), P_{t-1}\right)
$$

for all $s \geqslant t$. Moreover, for any sequence $\left.\left\langle p{ }_{s} \mid s\right\rangle t\right\rangle \in R_{+}^{T-t+1},(A 19)-(A 20)$ imply: $\left.\left.\pi_{T}\left(p_{S}\right)\right) \leqslant \pi_{T}\left(\varepsilon_{S}\right\rangle\right)$, where $\left\langle e_{S} \mid s \geqslant T\right\rangle \in \varepsilon_{t, T}$ is defined by $e_{s} \equiv \max \left(p(c), \min \left(p_{s}, P_{t-1}\right)\right)$. As a result :

(A22)

$$
\pi_{T}\left(\left(p_{S}\right)\right)<\pi_{T}\left(\left\langle p_{T, S}\right)\right)
$$

for any arbitrary $\left\langle p_{S} \mid s \geqslant t\right\rangle$. Let now the sequence $\left\langle p_{T, S} \mid t \leqslant s<T\right\rangle$ be 
extended into an infinite one by setting $\mathrm{p}_{T, s}=c$ for a $11 \mathrm{~s}>T$. Consider any fixed $s \geqslant t$ and let $T \geqslant s$ vary; the sequence $\left\langle p_{T, s} \mid T \geqslant s\right\rangle$ has values in the compact $\left[p(c), \max \left(p(c), p_{t-1}\right)\right]$, hence there exists a subsequence (p $\left.T_{T, S} \mid k \in N\right)$ converging to a limit $p_{s}^{*}$ with :

$$
(\forall s \geqslant t): \quad p(c)<p_{s}^{*} \leqslant \max \left(p(c), P_{t-1}\right)
$$

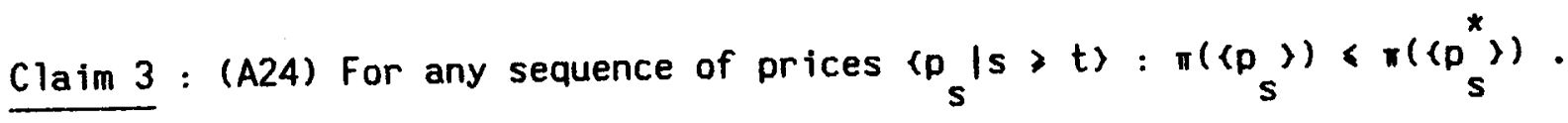
In other words, $\left\langle p_{s}^{*}\right.$ ) maximizes $\pi$. Fix $T>t$ large enough to have : (A25)

$$
\theta \lambda(p(c)-c) D(p(c)) \delta^{T-t+1} /[(1-\delta) \mu]<E / 2
$$

For all $s \geqslant t$ and $t \geqslant 0$ :

$$
\left(p_{s}-c\right) D\left(P_{s-\tau, s}\right)<\left(P_{s-\tau, s}-c\right) D\left(P P_{s-\tau, s}\right)<(p(c)-c) D(p(c))
$$

hence :

$$
\text { (A26) } \begin{aligned}
\pi\left(\left\langle p_{S}\right)\right) & =\pi_{T}\left(\left(p_{S}\right\rangle\right)+\theta \lambda \sum_{S=T+1}^{\infty} \delta^{s-t} \sum_{T=0}^{\infty}(1-\mu)^{T}\left(p_{S}-c\right) D\left(P P_{S-T, S}\right) \\
& <\pi_{T}\left(\left\langle p_{S}\right\rangle\right)+E / 2
\end{aligned}
$$

But, by definition of $\left\langle\mathrm{p}_{T, s}\right\rangle$ :

$$
{ }_{T}\left(\left\langle p_{S}\right\rangle\right)<\pi_{T}\left(\left\langle p_{T, S}\right)\right)=\pi_{T} \cdot\left(\left\langle p_{T, s}\right\}\right) \text { for all } T^{\prime} \geqslant T
$$

(because $p_{T, s}=c$ for $s>T$ ); similarly, by definition of $p_{T}, s$ :

$$
\pi_{T},\left(\left\langle p_{T, s}\right\rangle\right)<\pi_{T} \cdot\left(\left\langle p_{T^{\prime}, s^{\prime}}\right\rangle\right)=\pi\left(\left\langle p_{T^{\prime}, s^{\prime}}\right)<\pi_{T}\left(\left\langle p_{T^{\prime}, s^{\prime}}\right)+\epsilon / 2\right.\right.
$$

by (A26) applied with $p_{s}=p_{T^{\prime}, s}$ for all $s \geqslant t$. Thus, finally :

(A27)

$$
\left.\pi\left(p_{s}\right)\right)<\pi_{T}\left(\left(p_{T},, s^{\prime}\right)+E \quad\left(\forall T^{\prime}>T\right) .\right.
$$




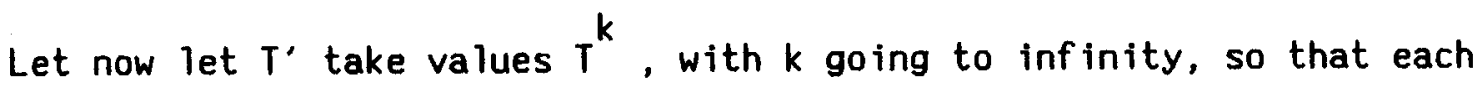
$p_{T^{\prime}, s}$ converges to $p_{s}^{*}$; since the above sum $\pi_{T}\left(\left\langle p_{T^{\prime}, s}\right)\right.$ involves only a fixed, finite number $T-t+1$ of such terms, it is licit to take limits to obtain :

$$
\pi\left(\left\langle p_{s}\right\rangle\right)<\pi \pi_{T}\left(\left\langle p_{s}^{*}\right\rangle\right)+E
$$

which holds for all $T$ large enough to have (A25). Finally, since for all $\mathrm{s}$, $p_{S}^{*}>p(c) \geqslant c$ by $(A 21)$, and since demand is always non-negative :

$$
\pi\left(\left\langle p^{S}\right\rangle\right)<\pi^{T}\left(\left\langle p^{*}\right\rangle\right)+E<\pi\left(\left\langle p^{*}\right\}\right)+E
$$

for all $\varepsilon>0$. Letting now $\varepsilon$ tend to zero concludes the proof. Q.E.D. Proof of Theorem IV.3:

The optimality of firms' strategies (given their beliefs and consumers' strategies) obtains by construction of $\left\langle p_{s}^{*}\left(H_{t}^{f}\right)\right\rangle$ in Lemma 2 . A consumer's problem is identical to what it was in Theorem IV.1, except that his effective discount rate is now $\delta \mu$. His beliefs over the cost types of firms and the prices charged in the past by those he did not visit have also been defined to be identical to what they were in that same theorem. By (35), firm's equilibrium strategies then induce the same (point) expectations over current and future price paths $P_{t, n}^{\sigma}(f)$. Each consumer's optimal strategy is therefore unchanged (except for the discount rate). Finally, the Bayesian property of beliefs also results from Theorem IV.1, since the equilibrium path as we 11 as beliefs along it are unchanged. Q.E.D. 